\title{
On the topology of solenoidal attractors of the cylinder
}

\section{Sur la topologie des attracteurs de type solenoïde du cylindre}

\author{
Rodrigo Bamón ${ }^{\mathrm{a}, *, 1}$, Jan Kiwi ${ }^{\mathrm{b}, 2}$, Juan Rivera-Letelier ${ }^{\mathrm{c}, 3}$, Richard Urzúa ${ }^{\mathrm{c}, 4}$ \\ a Departamento de Matemáticas, Facultad de Ciencias, Universidad de Chile, Casilla 653, Santiago, Chile \\ ${ }^{\mathrm{b}}$ Facultad de Matemáticas, Pontificia Universidad Católica, Casilla 306, Correo 22, Santiago, Chile \\ c Departamento de Matemáticas, Universidad Católica del Norte, Casilla 1280, Antofagasta, Chile
}

Received 10 March 2004; received in revised form 27 January 2005; accepted 18 March 2005

Available online 2 December 2005

\section{Abstract}

We study the dynamics of skew product endomorphisms acting on the cylinder $\mathbb{R} / \mathbb{Z} \times \mathbb{R}$, of the form

$$
(\theta, t) \mapsto(\ell \theta, \lambda t+\tau(\theta)),
$$

where $\ell \geqslant 2$ is an integer, $\lambda \in(0,1)$ and $\tau: \mathbb{R} / \mathbb{Z} \rightarrow \mathbb{R}$ is a continuous function. We are interested in topological properties of the global attractor $\Omega_{\lambda, \tau}$ of this map. Given $\ell$ and a Lipschitz function $\tau$, we show that the attractor set $\Omega_{\lambda, \tau}$ is homeomorphic to a closed topological annulus for all $\lambda$ sufficiently close to 1 . Moreover, we prove that $\Omega_{\lambda, \tau}$ is a Jordan curve for at most finitely many $\lambda \in(0,1)$.

These results rely on a detailed study of iterated "cohomological" equations of the form $\tau=\mathcal{L}_{\lambda_{1}} \mu_{1}, \mu_{1}=\mathcal{L}_{\lambda_{2}} \mu_{2}, \ldots$, where $\mathcal{L}_{\lambda} \mu=\mu \circ \mathbf{m}_{\ell}-\lambda \mu$ and $\mathbf{m}_{\ell}: \mathbb{R} / \mathbb{Z} \rightarrow \mathbb{R} / \mathbb{Z}$ denotes the multiplication by $\ell$ map. We show the following finiteness result: each Lipschitz function $\tau$ can be written in a canonical way as,

$$
\tau=\mathcal{L}_{\lambda_{1}} \circ \cdots \circ \mathcal{L}_{\lambda_{m}} \mu
$$

where $m \geqslant 0, \lambda_{1}, \ldots, \lambda_{m} \in(0,1]$ and the Lipschitz function $\mu$ satisfies $\mu \neq \mathcal{L}_{\lambda} \rho$ for every continuous function $\rho$ and every $\lambda \in(0,1]$.

C 2006 L'Association Publications de l'Institut Henri Poincaré. Published by Elsevier B.V. All rights reserved

\footnotetext{
* Corresponding author. Tel.: +(56)(2)6787295; fax: $+(56)(2) 2713882$.

E-mail addresses: rbamon@uchile.cl (R.Bamón), jkiwi@puc.cl (J. Kiwi), juanrive@ucn.cl (J. Rivera-Letelier), rurzua@ucn.cl (R. Urzúa).

1 Partially supported by Fondecyt \#1010865.

2 Partially supported by Fondecyt \#1020711.

3 Partially supported by Fundación Andes, PROSUL, and Fondecyt \#1070711.

4 Partially supported by Fondecyt \#1000047, DGICT-UCN and Fundación Andes.
} 


\section{Résumé}

On étudie la dynamique des produits croisés agissant sur le cylindre $\mathbb{R} / \mathbb{Z} \times \mathbb{R}$, de la forme

$$
(\theta, t) \mapsto(\ell \theta, \lambda t+\tau(\theta)),
$$

où $\ell \geqslant 2$ est un entier, $\lambda \in(0,1)$ et $\tau: \mathbb{R} / \mathbb{Z} \rightarrow \mathbb{R}$ est une fonction continue. On s'intéresse aux propriétés topologiques de l'attracteur global $\Omega_{\lambda, \tau}$ de cet endomorphisme. Étant donné $\ell$ et une fonction lipschitzienne $\tau$, on démontre que l'attracteur $\Omega_{\lambda, \tau}$ est homéomorphe à un anneau topologique pour tout $\lambda$ suffisamment proche de 1 . D'autre part, on démontre qu'il existe au plus un nombre fini de $\lambda \in(0,1)$ tels que l'attracteur $\Omega_{\lambda, \tau}$ soit une courbe de Jordan.

Ces résultats s'appuient sur une analyse détaillée des équations "cohomologiques" itérées : $\tau=\mathcal{L}_{\lambda_{1}} \mu_{1}, \mu_{1}=\mathcal{L}_{\lambda_{2}} \mu_{2}, \ldots$, où $\mathcal{L}_{\lambda} \mu=\mu \circ \mathbf{m}_{\ell}-\lambda \mu$ et $\mathbf{m}_{\ell}$ est l'application de multiplication par $\ell$ sur le cercle $\mathbb{R} / \mathbb{Z}$. On démontre le résultat de finitude suivant : toute fonction lipschitizenne $\tau$ s'écrit de façon canonique sous la forme

$$
\tau=\mathcal{L}_{\lambda_{1}} \circ \cdots \circ \mathcal{L}_{\lambda_{m}} \mu,
$$

où $m \geqslant 0, \lambda_{1}, \ldots, \lambda_{m} \in(0,1]$ et la fonction lispchitzienne $\mu$ satisfait $\mu \neq \mathcal{L}_{\lambda} \rho$ pour toute fonction continue $\rho$ et tout $\lambda \in(0,1]$. (C) 2006 L'Association Publications de l'Institut Henri Poincaré. Published by Elsevier B.V. All rights reserved

MSC: 37C70; 37D45; 37E99; 37G35

Keywords: Attractors; Endomorphisms

\section{Introduction}

In this paper we study the dynamics of skew product endomorphisms of the cylinder $\mathbb{R} / \mathbb{Z} \times \mathbb{R}$ of the form

$$
\begin{aligned}
A_{\lambda, \tau}: \mathbb{R} / \mathbb{Z} \times \mathbb{R} & \rightarrow \mathbb{R} / \mathbb{Z} \times \mathbb{R}, \\
(\theta, t) & \mapsto(\ell \theta, \lambda t+\tau(\theta)),
\end{aligned}
$$

where $\ell \geqslant 2$ is an integer, $\lambda \in(0,1)$ and $\tau: \mathbb{R} / \mathbb{Z} \rightarrow \mathbb{R}$ is a continuous function.

The non-wandering set $\Omega_{\lambda, \tau}$ of $A_{\lambda, \tau}$ is a global attractor of the dynamics of $A_{\lambda, \tau}$ : the forward orbit of every point in $\mathbb{R} / \mathbb{Z} \times \mathbb{R}$ converges to $\Omega_{\lambda, \tau}$ and $A_{\lambda, \tau}$ is transitive on $\Omega_{\lambda, \tau}$. In fact $A_{\lambda, \tau}$ is topologically semi-conjugate to a solenoidal map on $\Omega_{\lambda, \tau}$ (Section 2).

These maps where initially studied in [10], from a measure theoretical point of view. In that paper M. Tsujii showed that $A_{\lambda, \tau}$ has a unique physical measure and that the support of this measure is the attractor $\Omega_{\lambda, \tau}$. The main result of [10] is that, when $\lambda>\ell^{-1}$, for generic functions $\tau$ of class $C^{2}$ the unique physical measure of $A_{\lambda, \tau}$ is absolutely continuous with respect to Lebesgue measure.

The purpose of this paper is to study topological properties of the attractor sets $\Omega_{\lambda, \tau}$. Our main result is the following.

Theorem 1. Suppose that $\tau: \mathbb{R} / \mathbb{Z} \rightarrow \mathbb{R}$ is a non-constant Lipschitz function. Then the following hold:

(1) The set $\mathcal{J}_{\tau}=\left\{\lambda \in(0,1) \mid \Omega_{\lambda, \tau}\right.$ is a Jordan curve $\}$ is finite.

(2) There exists $\lambda_{0} \in(0,1)$ such that for all $\lambda \in\left[\lambda_{0}, 1\right)$ the attractor set $\Omega_{\lambda, \tau}$ is homeomorphic to an annulus.

For a given $\lambda \in(0,1)$, we characterize those functions $\tau$ for which $\Omega_{\lambda, \tau}$ is a Jordan curve in terms of the Fourier coefficients of $\tau$ (Theorem 2). From this characterization it follows easily that the set of those $\tau$ for which $\Omega_{\lambda, \tau}$ is a Jordan curve has infinite codimension in the space of all Lipschitz functions. 


\subsection{On the interior of the attractor}

When $\lambda<\ell^{-1}$, it is easy to see that $\Omega_{\lambda, \tau}$ has zero Lebesgue measure and hence empty interior. On the other hand, when $\lambda=\ell^{-1}$ we show the set $\Omega_{\lambda, \tau}$ is not homeomorphic to an annulus (Proposition 4.4). So $\lambda_{0}$ in part (2) of the theorem must be strictly larger than $\ell^{-1}$.

When $\lambda>\ell^{-1}$, Tsujii's result (mentioned above) implies that for most $\tau$ of class $C^{2}$, the attractor set $\Omega_{\lambda, \tau}$ has positive Lebesgue measure. Here we show examples of maps $A_{\lambda, \tau}$ for which the set $\Omega_{\lambda, \tau}$ has non-empty interior but it is not homeomorphic to an annulus (Proposition 7.4). In these examples $\lambda$ can be taken arbitrarily close to $\ell^{-1}$. Moreover we show that these examples are robust in the sense that any map $A: \mathbb{R} / \mathbb{Z} \times \mathbb{R} \rightarrow \mathbb{R} / \mathbb{Z} \times \mathbb{R}$ that is sufficiently (Lipschitz) close to $A_{\lambda, \tau}$ has the same properties. Here, $A$ is not necessarily a skew product map.

In a forthcoming paper we show that, when $\lambda>\ell^{-1}$, for most $\tau$ of class $C^{2}$ the set $\Omega_{\lambda, \tau}$ has non-empty interior.

\subsection{On the iterated cohomological equation}

Recall that a continuous function $\tau: \mathbb{R} / \mathbb{Z} \rightarrow \mathbb{R}$ is cohomologous to 0 if there is a continuous function $\mu$ such that

$$
\tau=\mathcal{L} \mu=\mu \circ \mathbf{m}_{\ell}-\mu,
$$

where $\mathbf{m}_{\ell}: \mathbb{R} / \mathbb{Z} \rightarrow \mathbb{R} / \mathbb{Z}$ is the multiplication by $\ell$ map. It is easy to see that in this case $\int_{\mathbb{R} / \mathbb{Z}} \tau=0$ and the function $\mu$ is unique up to an additive constant. For this reason we will assume that all the functions considered in the rest of the introduction have 0 mean.

Part (2) of the theorem is first proven in the case when $\tau$ is not cohomologous to 0 (Proposition 4.6). When $\tau=\mathcal{L} \mu$ is cohomologous to 0 , a direct computation shows that the maps $A_{\lambda, \tau}$ and $A_{\lambda, \mu}$ are conjugated by the homeomorphism $(\theta, t) \mapsto(\theta,(t+\mu) /(1-\lambda))$. So, if $\mu$ is not cohomologous to 0 we reduce to the first case. By induction, if for some positive integer $n$ there is a continuous function $\mu: \mathbb{R} / \mathbb{Z} \rightarrow \mathbb{R}$ that is not cohomologous to 0 and such that $\tau=\mathcal{L}^{n} \mu$, then we reduce to the first case.

We complete the proof of part (2) of the theorem by showing that a non-constant Lipschitz function cannot be infinitely cohomologous to 0 . More precisely, we show that if $\tau$ is Lipschitz, then the integer $n$ above is bounded by a constant depending only on the Fourier coefficients of $\tau$ (Corollary 5.7).

Problem 1. Is there a non-constant continuous function that is infinitely cohomologous to 0 ?

\subsection{Cohomological operators}

For $\lambda \in(0,1]$ it is interesting to consider the linear operators $\mathcal{L}_{\lambda}$ defined by $\mathcal{L}_{\lambda} \mu=\lambda \mu-\mu \circ \mathbf{m}_{\ell}$, so that $\mathcal{L}_{1}=\mathcal{L}$. For $\lambda_{0} \in(0,1)$ and $\tau$ (Lipschitz) continuous we show that $\Omega_{\lambda_{0}, \tau}$ is a Jordan curve if and only if there exists a (Lipschitz) continuous function $\mu$ such that $\tau=\mathcal{L}_{\lambda_{0}} \mu$ (Proposition 3.1). In that case, for every $\lambda \in(0,1)$ different from $\lambda_{0}$, the maps $A_{\lambda, \tau}$ and $A_{\lambda, \mu}$ are conjugate (Lemma 5.8).

We show that each Lipschitz function $\tau$ can be written in a canonical way as

$$
\tau=\mathcal{L}_{\lambda_{1}} \circ \cdots \circ \mathcal{L}_{\lambda_{m}} \mu
$$

where the function $\mu$ satisfies $\int_{\mathbb{R} / \mathbb{Z}} \mu=0$ and $\mu \neq \mathcal{L}_{\lambda} \rho$ for every $\lambda \in(0,1]$ and every continuous function $\rho$ (here there might be repetitions among $\lambda_{1}, \ldots, \lambda_{m}$ ). This implies part (1) of the theorem. Note that such a function $\mu$ is such that for every $\lambda \in(0,1)$ the set $\Omega_{\lambda, \mu}$ is not a Jordan curve and for every $\lambda \in(0,1)$ different from the $\lambda_{i}$, the maps $A_{\lambda, \tau}$ and $A_{\lambda, \mu}$ are conjugate (Theorem 3).

Problem 2. For $\ell=2$, let $\mu$ be a non-constant Lipschitz function such that $\mu \neq \mathcal{L}_{\lambda} \rho$ for every $\lambda \in(0,1)$ and every continuous function $\rho$. Is there $\lambda_{0} \in(0,1)$ such that $\Omega_{\lambda, \mu}$ is homeomorphic to an annulus if and only if $\lambda \geqslant \lambda_{0}$ ? 


\subsection{Are there periodic points in the boundary?}

The upper (resp. lower) boundary of the attractor is by definition the graph of the function

$$
\begin{aligned}
& \rho^{+}(\theta)=\sup \left\{t \mid(\theta, t) \in \Omega_{\lambda, \tau}\right\} \\
& \text { (resp. } \left.\rho^{-}(\theta)=\inf \left\{t \mid(\theta, t) \in \Omega_{\lambda, \tau}\right\}\right) .
\end{aligned}
$$

These functions are continuous and characterized by the functional equations

$$
\begin{aligned}
& \rho^{+}(\theta)=\max \left\{\lambda \rho^{+}\left(\theta^{\prime}\right)+\tau\left(\theta^{\prime}\right) \mid \theta^{\prime} \in \mathbf{m}_{\ell}^{-1}(\theta)\right\}, \\
& \rho^{-}(\theta)=\min \left\{\lambda \rho^{-}\left(\theta^{\prime}\right)+\tau\left(\theta^{\prime}\right) \mid \theta^{\prime} \in \mathbf{m}_{\ell}^{-1}(\theta)\right\} .
\end{aligned}
$$

Moreover, when $\tau$ is Lipschitz the functions $\rho^{+}$and $\rho^{-}$are also Lipschitz.

Conjecture. For each $\lambda \in(0,1)$ there is an open and dense set of functions $\tau$ of class $C^{1}$, such that the following properties hold.

1. The upper (resp. lower) boundary of $\Omega_{\lambda, \tau}$ contains a finite number of periodic orbits of $A_{\lambda, \tau}$.

2. The upper (resp. lower) boundary of $\Omega_{\lambda, \tau}$ is formed by a finite number of pieces of the unstable manifolds of the periodic orbits that it contains.

In particular the upper and lower boundaries are $C^{1}$ by parts.

For a given $\lambda \in(0,1)$ and a continuous function $\tau: \mathbb{R} / \mathbb{Z} \rightarrow \mathbb{R}$ consider the closed set

$$
D^{+}=\left\{\theta \in \mathbb{R} / \mathbb{Z} \mid \lambda \rho^{+}(\theta)+\tau(\theta)=\rho^{+}(\ell \theta)\right\} .
$$

It follows from the functional equation of $\rho^{+}$that $\mathbf{m}_{\ell}\left(D^{+}\right)=\mathbb{R} / \mathbb{Z}$, so the maximal invariant set

$$
K^{+}=\left\{\theta \in D^{+} \mid \mathbf{m}_{\ell}^{n}(\theta) \in D^{+} \text {for } n \geqslant 1\right\}
$$

is non-empty and compact. Part 1 of the conjecture implies that $K^{+}$contains a finite number of periodic orbits and part 2 implies that $K^{+}$is finite.

The above conjecture is somewhat similar to the conjecture that, for generic expanding endomorphisms of the circle $f$, there is a unique measure $\mu$ minimizing the integral $\int_{\mathbb{R} / \mathbb{Z}} \ln f^{\prime} \mathrm{d} \mu$ and that this measure has finite support, see $[1,2]$ and references therein.

\subsection{Notes and references}

Our original motivation to study the dynamics of the skew product maps $A_{\lambda, \tau}$ was the family of maps $f_{\lambda, c}$ considered in Subsection 7.1.

Similar skew products of the cylinder where studied by M. Viana [12]. M. Tsujii [11] extended the results of [10] and [12] to general partially hyperbolic endomorphisms on surfaces. The structural stability of Axiom A endomorphisms has been studied in [9]. Piecewise affine endomorphisms having an attractor set with non-empty interior were studied in [3].

After this article was written we found out that the skew product maps considered here were studied in [6] and [4], see also Appendix 3 of [8]. In [4] there are some examples of maps $A_{\lambda, \tau}$ whose attractor set $\Omega_{\lambda, \tau}$ has non-empty interior.

Some results in this paper are closely related to "normal forms" in "ergodic optimization" (e.g. see [5] and references therein). For example, given a function $\tau: \mathbb{R} / \mathbb{Z} \rightarrow \mathbb{R}$ the study of the solutions $\rho^{+}$of the equation

$$
\rho^{+}(\theta)=\max \left\{\lambda \rho^{+}\left(\theta^{\prime}\right)+\tau\left(\theta^{\prime}\right) \mid \theta^{\prime} \in \mathbf{m}_{\ell}^{-1}(\theta)\right\}
$$


as $\lambda \rightarrow 1$ leads to the existence of a normal form of $\tau$. It is remarkable that in our context the function $\rho^{+}$has the clear geometrical interpretation as the upper boundary of an attractor set.

\subsection{Outline}

Let us now describe the structure of the paper.

Section 2 starts giving several equivalent characterizations of the set $\Omega_{\lambda, \tau}$. Then, in Subsection 2.1, we show that the semiconjugacy between the dynamics induced by the multiplication by $\ell$ map on the solenoid $\mathcal{S}$ and the dynamics of $A_{\lambda, \tau}$ on $\Omega_{\lambda, \tau}$ can be written in a fairly explicit manner. Also, we endow $\mathcal{S}$ with an adapted metric dist $_{\lambda}$ which makes this semiconjugacy a Lipschitz function, provided that $\tau$ is Lipschitz. Then it naturally follows that the upper and lower boundaries of the attractor $\Omega_{\lambda, \tau}$ are Lipschitz graphs when $\tau$ is Lipschitz (Subsection 2.2).

Section 3 is devoted to study Jordan curve attractors. We characterize them and show, among other results, that $\Omega_{\lambda, \tau}$ is a Jordan curve if and only if the functional equation $\mu \circ \mathbf{m}_{\ell}-\lambda \mu=\tau$ has a continuous solution (Proposition 3.1). This allow us to show, in Subsection 3.2, that the set of continuous $\tau$ (with absolutely convergent Fourier series) such that $\Omega_{\lambda, \tau}$ is a Jordan curve has infinite codimension (Theorem 2).

The main result in Section 4 is that, for $\lambda$ sufficiently close to 1 , the attractor $\Omega_{\lambda, \tau}$ is a closed topological annulus provided that $\tau$ is Hölder, not cohomologous to 0 and with 0 mean (Proposition 4.6). This section starts with general results about annular attractors. In particular, we show that if the image of the upper boundary of $\Omega_{\lambda, \tau}$ is above in the cylinder $\mathbb{R} / \mathbb{Z} \times \mathbb{R}$ than the image of the lower boundary, then $\Omega_{\lambda, \tau}$ is homeomorphic to a closed annulus (Lemma 4.1). Also, we establish that for $\lambda \leqslant 1 / \ell$, the attractor $\Omega_{\lambda, \tau}$ cannot be an annulus (Proposition 4.4). Then, in Subsection 4.1, under the above assumptions for $\tau$ we find periodic orbits $\mathcal{O}^{ \pm}$in the circle so that the corresponding orbits in $\Omega_{\lambda, \tau} \subset \mathbb{R} / \mathbb{Z} \times \mathbb{R}$ have $\mathbb{R}$ coordinates tending to $\pm \infty$ as $\lambda \rightarrow 1$. From this we deduce that the image of the upper boundary is higher up in $\mathbb{R} / \mathbb{Z} \times \mathbb{R}$ than the image of the lower boundary, when $\lambda$ is sufficiently close to 1 , and therefore that $\Omega_{\lambda, \tau}$ is an annulus.

Sections 3 and 4 lead us to study in more detail the operators $\mathcal{L}_{\lambda} \mu=\mu \circ \mathbf{m}_{\ell}-\lambda \mu$ with $\lambda \in(0,1]$. For our purpose the natural domain of the operators $\mathcal{L}_{\lambda}$ is the space of Lipschitz functions. In Section 5 we start by proving some general facts about these linear operators and relating them to conjugacy classes of maps of the form $A_{\lambda, \tau}$ (Lemmas 5.2 and 5.8). As mentioned in Subsection 1.2 this forces us to study iterated equations of the form $\mathcal{L}_{\lambda_{1}} \mu_{1}=\tau, \mathcal{L}_{\lambda_{2}} \mu_{2}=\mu_{1}, \ldots$ An important feature of the operators $\mathcal{L}_{\lambda}$ is that they do not increase the best Lipschitz constant for $\mu$ (Lemma 5.4) which implies that solutions $\mu_{n}$ of the iterated equations are uniformly Lipschitz. Then we show that the above equations have the effect of increasing the Fourier coefficients of $\mu_{n}$ as $n$ increases and establish our Main Lemma which states that, given a Lipschitz function $\tau$ there exists a finite collection $0<\lambda_{1}, \ldots, \lambda_{m} \leqslant 1$ (maybe with repetitions) and a (Lipschitz) continuous function $\mu$ such that $\mathcal{L}_{\lambda_{1}} \circ \cdots \circ \mathcal{L}_{\lambda_{m}} \mu=\tau$ and $\mathcal{L}_{\lambda} \rho \neq \mu$ for all continuous $\rho$ (see Lemma 5.6 and the Main Lemma). Theorem 1 and its stronger version Theorem 3 follow immediately from our Main Lemma.

In Section 6 we start by appropriately defining the attractor set and the upper and lower boundaries for arbitrary maps (not necessarily skew products) which are close to $A_{\lambda, \tau}$ and state that the upper and lower boundaries of $\Omega_{\lambda, \tau}$ vary continuously in the $C^{0}$ topology under Lipschitz perturbations of $A_{\lambda, \tau}$ (Proposition 6.2). To prove this we pass to the universal cover $\mathbb{R}^{2}$ of $\mathbb{R} / \mathbb{Z} \times \mathbb{R}$ and, in Subsection 6.1, examine the action of Lipschitz maps from $\mathbb{R}^{2}$ into $\mathbb{R}^{2}$ on the graphs of Lipschitz functions. In Subsection 6.2, motivated by the fact that the upper and lower boundaries of $\Omega_{\lambda, \tau}$ are the graphs of functions $\rho^{ \pm}: \mathbb{R} / \mathbb{Z} \rightarrow \mathbb{R}$ which satisfy certain functional equations we show that, under certain conditions, the upper and lower boundaries of the attractor of maps $F$ of the cylinder are fixed points of operators $\mathcal{T}_{F}^{ \pm}$which act on Lipschitz functions. The definition and properties of $\mathcal{T}_{F}^{ \pm}$rely on the work of Subsection 6.1. At the end of Section 6 we prove the above mentioned continuity of the upper and lower boundaries of the attractor.

The last section, Section 7, contains two examples. The first example consists of an application of our results to the study of a family $f_{\lambda, c}$ of endomorphisms of $\mathbb{C}^{*}=\mathbb{C} \backslash\{0\}$ where $\lambda \in(0,1)$ and $c \in \mathbb{C}^{*}$. Here $f_{\lambda, c}=f_{\lambda, 0}+c$ where $f_{\lambda, 0}$ acts as angle doubling on the arguments of $z \in \mathbb{C}^{*}$ and as an affine contraction of factor $\lambda$ in the 
radial direction. Thus, $f_{\lambda, c}$ is closely related to the extensively studied quadratic family $Q_{c}(z)=z^{2}+c$ where the $|z| \mapsto|z|^{2}$ action of $Q_{0}$ in the radial direction has been substituted by an affine contraction. We show that for $\lambda$ sufficiently close to 1 and for $|c|$ sufficiently small, the attractor of $f_{\lambda, c}$ is an annulus. The second example shows that given $\lambda>1 / \ell$ there exist skew product endomorphisms $A_{\lambda, \tau}$ such that the attractors $\Omega_{\lambda, \tau}$ have non-empty interior and are not an annulus. This example is robust under Lipschitz perturbations.

\section{Preliminaries}

Throughout this section, unless otherwise stated, $\tau: \mathbb{R} / \mathbb{Z} \rightarrow \mathbb{R}$ is a continuous function and $\lambda \in(0,1)$. We start by showing that $\Omega_{\lambda, \tau}$ is a global attractor for the dynamics of $A_{\lambda, \tau}$ and giving several equivalent characterizations of this set. As usual $\|\tau\|_{\infty}=\sup \{\tau(\theta) \mid \theta \in \mathbb{R} / \mathbb{Z}\}$.

Lemma 2.1. Let $\Omega_{\lambda, \tau}$ be the non-wandering set of $A_{\lambda, \tau}: \mathbb{R} / \mathbb{Z} \times \mathbb{R} \rightarrow \mathbb{R} / \mathbb{Z} \times \mathbb{R}$.

(1) If $U_{0}=\mathbb{R} / \mathbb{Z} \times\left(-T_{0}, T_{0}\right)$ for some $T_{0}$ such that $(1-\lambda) T_{0}>\|\tau\|_{\infty}$, then

$$
A_{\lambda, \tau}\left(\overline{U_{0}}\right) \subset U_{0} \quad \text { and } \quad \Omega_{\lambda, \tau}=\bigcap_{n \geqslant 0} A_{\lambda, \tau}^{n}\left(U_{0}\right) .
$$

(2) $\Omega_{\lambda, \tau}$ is the set of all $(\theta, t) \in \mathbb{R} / \mathbb{Z} \times \mathbb{R}$ with a bounded infinite backward orbit (i.e., there exists $C>0$ and $\left\{\left(\theta_{n}, t_{n}\right)\right\}_{n} \geqslant 1$ such that $A_{\lambda, \tau}^{n}\left(\theta_{n}, t_{n}\right)=(\theta, t)$ and $\left|t_{n}\right| \leqslant C$ for all $\left.n \geqslant 1\right)$.

(3) $\Omega_{\lambda, \tau}$ is the closure of the set formed by the periodic points of $A_{\lambda, \tau}$.

Proof. Denote by Per the set of periodic points of $A_{\lambda, \tau}$ and by $B$ the set of points in $\mathbb{R} / \mathbb{Z} \times \mathbb{R}$ which have a bounded infinite backward orbit. We will show that:

$$
\overline{\operatorname{Per}} \subset \Omega_{\lambda, \tau} \subset \bigcap_{n \geqslant 0} A_{\lambda, \tau}^{n}\left(U_{0}\right) \subset B \subset \overline{\operatorname{Per}} .
$$

The inclusions $\overline{\operatorname{Per}} \subset \Omega_{\lambda, \tau}$ and $\bigcap_{n \geqslant 0} A_{\lambda, \tau}^{n}\left(U_{0}\right) \subset B$ are clear.

Let $T_{0}$ be such that $(1-\lambda) T_{0}>\|\tau\|_{\infty}$. Since

$$
|\lambda t+\tau(\theta)| \leqslant \lambda T_{0}+\|\tau\|_{\infty}<\lambda T_{0}+(1-\lambda) T_{0}=T_{0}
$$

for all $|t| \leqslant T_{0}$, it follows that $A_{\lambda, \tau}\left(\overline{U_{0}}\right) \subset U_{0}$.

Note that if $\left(\theta_{n}, t_{n}\right)=A_{\lambda, \tau}^{n}(\theta, t)$, then $\theta_{n}=\ell^{n} \theta$ and

$$
t_{n}=\lambda^{n} t+\lambda^{n-1} \tau(\theta)+\lambda^{n-2} \tau(\ell \theta)+\cdots+\tau\left(\ell^{n-1} \theta\right) .
$$

Therefore,

$$
\left|t_{n}\right| \leqslant \frac{1-\lambda^{n}}{1-\lambda}\|\tau\|_{\infty}+\lambda^{n}|t|
$$

Hence, for all $(\theta, t) \in \mathbb{R} / \mathbb{Z} \times \mathbb{R}$ there exists $n$ such that $A_{\lambda, \tau}^{n}(\theta, t) \in U_{0}$.

Now we show that $\Omega_{\lambda, \tau} \subset \bigcap_{n \geqslant 0} A_{\lambda, \tau}^{n}\left(U_{0}\right)$. In fact, suppose that for some $m \geqslant 0$ we have that $(\theta, t) \notin A_{\lambda, \tau}^{m}\left(\bar{U}_{0}\right)$ and consider $n$ such that $(\theta, t)$ belongs to the open set $V=A_{\lambda, \tau}^{-n}\left(U_{0}\right) \backslash A_{\lambda, \tau}^{m}\left(\bar{U}_{0}\right)$. Then, for every $M \geqslant m$ we have that $V$ is disjoint from $A_{\lambda, \tau}^{n+M}(V)$. It follows that $(\theta, t)$ does not belong to the non-wandering set $\Omega_{\lambda, \tau}$ of $A_{\lambda, \tau}$.

To finish the proof of the lemma we consider a neighborhood $U$ of a point $(\theta, t)$ with bounded infinite backward orbit and proceed to show that $U$ contains a periodic point. Let $\left\{\left(\theta_{n}, t_{n}\right)\right\}_{n} \geqslant 1$ and $C>0$ be such that $A_{\lambda, \tau}^{n}\left(\theta_{n}, t_{n}\right)=$ $(\theta, t)$ and $\left|t_{n}\right| \leqslant C$ for all $n \geqslant 1$. There exist $n \geqslant 1$ and an open interval $I \subset \mathbb{R} / \mathbb{Z}$ around $\theta_{n}$ such that the rectangle 
$R=I \times\left[-T_{0}, T_{0}\right]$ maps into $U$ under $A_{\lambda, \tau}^{n}$. Since there exists $\theta^{\prime} \in I$ periodic under multiplication by $\ell$, say of period $m$, we have that $A_{\lambda, \tau}^{m}$ restricted to $\left\{\theta^{\prime}\right\} \times\left[-T_{0}, T_{0}\right]$ is a contraction. It follows that $A_{\lambda, \tau}$ has a periodic point in $\left\{\theta^{\prime}\right\} \times\left[-T_{0}, T_{0}\right] \subset R$ and therefore in $U$.

\subsection{The solenoid and the semiconjugacy}

Throughout the paper multiplication by $\ell$ in the circle will be denoted by $\mathbf{m}_{\ell}: \mathbb{R} / \mathbb{Z} \rightarrow \mathbb{R} / \mathbb{Z}$. For each $\lambda \in(0,1)$ we endow the solenoid:

$$
\mathcal{S}:=\left\{\bar{\theta}=\left(\theta_{k}\right) \in(\mathbb{R} / \mathbb{Z})^{\mathbb{N} \cup\{0\}} \mid \mathbf{m}_{\ell}\left(\theta_{k+1}\right)=\theta_{k} \text { for all } k \geqslant 0\right\}
$$

with the adapted metric

$$
\operatorname{dist}_{\lambda}\left(\left(\theta_{k}\right),\left(\theta_{k}^{\prime}\right)\right)=\sum_{k \geqslant 0} \lambda^{k} \operatorname{dist}_{\mathbb{R} / \mathbb{Z}}\left(\theta_{k}, \theta_{k}^{\prime}\right),
$$

where dist $\mathbb{R} / \mathbb{Z}$ denotes the projection of the standard metric of $\mathbb{R}$ onto $\mathbb{R} / \mathbb{Z}$. The dynamics of multiplication by $\ell$ induces:

$$
\begin{aligned}
\mathcal{M}_{\ell}: \mathcal{S} & \rightarrow \mathcal{S}, \\
\left(\theta_{k}\right)_{k \geqslant 0} & \mapsto\left(\ell \theta_{0}, \theta_{0}, \theta_{1}, \ldots\right) .
\end{aligned}
$$

We will show that the dynamics of $\mathcal{M}_{\ell}: \mathcal{S} \rightarrow \mathcal{S}$ semi-conjugates to that of $A_{\lambda, \tau}: \Omega_{\lambda, \tau} \rightarrow \Omega_{\lambda, \tau}$. Thus, the attractor $\Omega_{\lambda, \tau}$ is in this sense a solenoidal attractor. To write an explicit formula for the semiconjugacy from $\mathcal{S}$ onto $\Omega_{\lambda, \tau}$ we need the following definition.

Definition 2.2. Given a continuous function $\tau: \mathbb{R} / \mathbb{Z} \rightarrow \mathbb{R}$ and $\lambda \in(0,1)$ we define $t_{\lambda}: \mathcal{S} \rightarrow \mathbb{R}$ by

$$
t_{\lambda}(\bar{\theta})=\tau\left(\theta_{1}\right)+\lambda \tau\left(\theta_{2}\right)+\lambda^{2} \tau\left(\theta_{3}\right)+\cdots .
$$

Note that $t_{\lambda}$ is continuous. Under the assumption that $\tau$ is a Lipschitz function we will show that $t_{\lambda}$ is also Lipschitz. In order to make the statements precise we introduce some notation.

Notation 2.3. Consider two metric spaces $\left(X, \rho_{X}\right),\left(Y, \rho_{Y}\right)$. Given a Lipschitz map $f: X \rightarrow Y$ the best Lipschitz constant for $f$

$$
\sup _{a \neq b} \frac{\rho_{Y}(f(a), f(b))}{\rho_{X}(a, b)}
$$

is denoted by $\|f\|_{L}$ and if $C \geqslant\|f\|_{L}$, we say that $f$ is a $C$-Lipschitz map.

Lemma 2.4. If $\lambda \in(0,1)$ and $\tau: \mathbb{R} / \mathbb{Z} \rightarrow \mathbb{R}$ is a Lipschitz function, then $t_{\lambda}$ is a $\left(\lambda^{-1}\|\tau\|_{L}\right)$-Lipschitz function from $\left(\mathcal{S}\right.$, dist $\left._{\lambda}\right)$ to $\mathbb{R}$.

Proof. For any $\bar{\theta}=\left(\theta_{k}\right)$ and $\bar{\theta}^{\prime}=\left(\theta_{k}^{\prime}\right)$ in $\mathcal{S}$ we have

$$
\begin{aligned}
\left|t_{\lambda}(\bar{\theta})-t_{\lambda}\left(\bar{\theta}^{\prime}\right)\right| & \leqslant \sum_{k \geqslant 1} \lambda^{k-1}\left|\tau\left(\theta_{k}\right)-\tau\left(\theta_{k}^{\prime}\right)\right| \leqslant\|\tau\|_{L} \cdot \sum_{k \geqslant 1} \lambda^{k-1} \operatorname{dist}_{\mathbb{R} / \mathbb{Z}}\left(\theta_{k}, \theta_{k}^{\prime}\right) \\
& =\lambda^{-1}\|\tau\|_{L}\left(\operatorname{dist}_{\lambda}\left(\bar{\theta}, \bar{\theta}^{\prime}\right)-\operatorname{dist}_{\mathbb{R} / \mathbb{Z}}\left(\theta_{0}, \theta_{0}^{\prime}\right)\right) \leqslant \lambda^{-1}\|\tau\|_{L} \operatorname{dist}_{\lambda}\left(\bar{\theta}, \bar{\theta}^{\prime}\right) .
\end{aligned}
$$

That is, $t_{\lambda}$ is $\lambda^{-1}\|\tau\|_{L}$-Lipschitz. 
Proposition 2.5. Given $\lambda \in(0,1)$ and a continuous function $\tau: \mathbb{R} / \mathbb{Z} \rightarrow \mathbb{R}$, let

$$
\begin{gathered}
h_{\lambda}: \mathcal{S} \rightarrow \mathbb{R} / \mathbb{Z} \times \mathbb{R}, \\
\bar{\theta}=\left(\theta_{k}\right) \mapsto\left(\theta_{0}, t_{\lambda}(\bar{\theta})\right) .
\end{gathered}
$$

Then $h_{\lambda}$ is a continuous semiconjugacy from $\mathcal{S}$ onto $\Omega_{\lambda, \tau}$. That is, $h_{\lambda}: \mathcal{S} \rightarrow \Omega_{\lambda, \tau}$ is onto and $A_{\lambda, \tau} \circ h_{\lambda}=h_{\lambda} \circ \mathcal{M}_{\ell}$. Moreover, $h_{\lambda}:\left(\mathcal{S}\right.$, dist $\left._{\lambda}\right) \rightarrow \Omega_{\lambda, \tau}$ is Lipschitz whenever $\tau: \mathbb{R} / \mathbb{Z} \rightarrow \mathbb{R}$ is Lipschitz.

Proof. That $A_{\lambda, \tau} \circ h_{\lambda}=h_{\lambda} \circ \mathcal{M}_{\ell}$ is a straightforward computation. We must show that $h_{\lambda}(\mathcal{S})=\Omega_{\lambda, \tau}$. Since $h_{\lambda}(\mathcal{S})$ is bounded and forward invariant (i.e., $A_{\lambda, \tau}\left(h_{\lambda}(\mathcal{S})\right)=h_{\lambda}(\mathcal{S})$ ), by Lemma 2.1, we have that $h(\mathcal{S}) \subset \Omega_{\lambda, \tau}$. Now if $\left(\theta_{0}, t_{0}\right) \in \Omega_{\lambda, \tau}$, then there exists a bounded backward orbit $\left\{\left(\theta_{n}, t_{n}\right)\right\}_{n} \geqslant 0$. Therefore, $\bar{\theta}=\left(\theta_{n}\right) \in \mathcal{S}$ and $t_{0}=t_{\lambda}(\bar{\theta})$. Hence, $\left(\theta_{0}, t_{0}\right)=h_{\lambda}(\bar{\theta}) \in h_{\lambda}(\mathcal{S})$. By the previous lemma, $h_{\lambda}$ is Lipschitz, if $\tau: \mathbb{R} / \mathbb{Z} \rightarrow \mathbb{R}$ is a Lipschitz function.

\subsection{Upper and lower boundaries of the attractor}

The attractor $\Omega_{\lambda, \tau}$ lies in between the graph of two functions which we call the upper and lower boundaries of $\Omega_{\lambda, \tau}$. More precisely:

Definition 2.6. Let

$$
\begin{aligned}
& \rho^{+}(\theta)=\sup \left\{t \mid(\theta, t) \in \Omega_{\lambda, \tau}\right\}, \\
& \rho^{-}(\theta)=\inf \left\{t \mid(\theta, t) \in \Omega_{\lambda, \tau}\right\} .
\end{aligned}
$$

We say that $\partial_{ \pm} \Omega_{\lambda, \tau}=\left\{\left(\theta, \rho_{ \pm}(\theta)\right) \mid \theta \in \mathbb{R} / \mathbb{Z}\right\}$ are the upper and lower boundaries of $\Omega_{\lambda, \tau}$, respectively.

Since $A_{\lambda, \tau}\left(\Omega_{\lambda, \tau}\right)=\Omega_{\lambda, \tau}$ and $A_{\lambda, \tau}$ is locally orientation preserving, it follows that

$$
\begin{aligned}
& \rho^{+}(\theta)=\max \left\{\lambda \rho^{+}\left(\theta^{\prime}\right)+\tau\left(\theta^{\prime}\right) \mid \theta^{\prime} \in \mathbf{m}_{\ell}^{-1}(\theta)\right\}, \\
& \rho^{-}(\theta)=\min \left\{\lambda \rho^{-}\left(\theta^{\prime}\right)+\tau\left(\theta^{\prime}\right) \mid \theta^{\prime} \in \mathbf{m}_{\ell}^{-1}(\theta)\right\} .
\end{aligned}
$$

For $\tau$ Lipschitz, the upper and lower boundaries are Lipschitz graphs. In general, for $\tau$ of class $C^{\infty}$ or even real analytic, the upper and lower boundaries are not $C^{1}$.

Lemma 2.7. If $\tau: \mathbb{R} / \mathbb{Z} \rightarrow \mathbb{R}$ is Lipschitz, then $\rho^{ \pm}: \mathbb{R} / \mathbb{Z} \rightarrow \mathbb{R}$ are $\left(\|\tau\|_{L} /(\ell-\lambda)\right)$-Lipschitz maps.

Proof. Consider $\theta_{0}, \theta_{0}^{\prime} \in \mathbb{R} / \mathbb{Z}$. Let $\bar{\theta}=\left(\theta_{k}\right) \in \mathcal{S}$ be such that $t_{\lambda}(\bar{\theta})=\rho^{+}\left(\theta_{0}\right)$. There exists $\bar{\theta}^{\prime}=\left(\theta_{k}^{\prime}\right) \in \mathcal{S}$ such that $\operatorname{dist}_{\mathbb{R} / \mathbb{Z}}\left(\theta_{k}^{\prime}, \theta_{k}\right)=\ell^{-k} \operatorname{dist}_{\mathbb{R} / \mathbb{Z}}\left(\theta_{0}^{\prime}, \theta_{0}\right)$ and therefore,

$$
\operatorname{dist}_{\lambda}\left(\bar{\theta}, \bar{\theta}^{\prime}\right)=\frac{\ell}{\ell-\lambda} \operatorname{dist}_{\mathbb{R} / \mathbb{Z}}\left(\theta_{0}, \theta_{0}^{\prime}\right) .
$$

Then, by (2):

$$
\begin{aligned}
\rho^{+}\left(\theta_{0}^{\prime}\right) \geqslant t_{\lambda}\left(\bar{\theta}^{\prime}\right) & =t_{\lambda}\left(\bar{\theta}^{\prime}\right)-t_{\lambda}(\bar{\theta})+t_{\lambda}(\bar{\theta}) \geqslant-C\left(\operatorname{dist}_{\lambda}\left(\bar{\theta}, \bar{\theta}^{\prime}\right)-\operatorname{dist}_{\mathbb{R} / \mathbb{Z}}\left(\theta_{0}, \theta_{0}^{\prime}\right)\right)+t_{\lambda}(\bar{\theta}) \\
& =-C\left(\frac{\ell}{\ell-\lambda}-1\right) \operatorname{dist}_{\lambda}\left(\bar{\theta}, \bar{\theta}^{\prime}\right)+t_{\lambda}(\bar{\theta})=\rho^{+}\left(\theta_{0}\right)-C \frac{\lambda}{\ell-\lambda} \operatorname{dist}_{\lambda}\left(\bar{\theta}, \bar{\theta}^{\prime}\right)
\end{aligned}
$$

where $C=\lambda^{-1}\|\tau\|_{L}$ is a Lipschitz constant for $t_{\lambda}$ (see Lemma 2.4). It follows that $\rho^{+}: \mathbb{R} / \mathbb{Z} \rightarrow \mathbb{R}$ is Lipschitz with the appropriate constant. For $\rho^{-}: \mathbb{R} / \mathbb{Z} \rightarrow \mathbb{R}$ a similar argument can be applied. 


\section{Jordan curve attractors}

Observe that the equator $\{0\} \times \mathbb{R} / \mathbb{Z}$ of the cylinder is the attractor of the map $A_{\lambda, 0}$.

\subsection{Characterization}

The next proposition characterizes Jordan curve attractors.

Proposition 3.1. Let $\tau: \mathbb{R} / \mathbb{Z} \rightarrow \mathbb{R}$ be a function of class $\mathcal{C}$ where $\mathcal{C}$ is either the Lipschitz class, or the $C^{r}$ class for some $r \in[0, \infty] \cup\{\omega\}$.

Then the following are equivalent:

(1) $A_{\lambda, \tau}$ is topologically conjugate to $A_{\lambda, 0}$.

(1') $A_{\lambda, \tau}$ is $\mathcal{C}$-conjugate to $A_{\lambda, 0}$.

(2) $\Omega_{\lambda, \tau} \subset \mathbb{R} / \mathbb{Z} \times \mathbb{R}$ is the graph of a continuous function $\mu: \mathbb{R} / \mathbb{Z} \rightarrow \mathbb{R}$.

(2') $\Omega_{\lambda, \tau} \subset \mathbb{R} / \mathbb{Z} \times \mathbb{R}$ is the graph of a function $\mu: \mathbb{R} / \mathbb{Z} \rightarrow \mathbb{R}$ of class $\mathcal{C}$.

(3) The functional equation $\mu \circ \mathbf{m}_{\ell}-\lambda \mu=\tau$ has a continuous solution $\mu: \mathbb{R} / \mathbb{Z} \rightarrow \mathbb{R}$.

(3') The functional equation $\mu \circ \mathbf{m}_{\ell}-\lambda \mu=\tau$ has a solution $\mu: \mathbb{R} / \mathbb{Z} \rightarrow \mathbb{R}$ of class $\mathcal{C}$.

(4) $\rho^{+}(\theta)=\rho^{-}(\theta)$ for all $\theta \in \mathbb{R} / \mathbb{Z}$.

(5) $\rho^{+}(\theta)=\rho^{-}(\theta)$ for some $\theta \in \mathbb{R} / \mathbb{Z}$.

(6) $\Omega_{\lambda, \tau}$ is a Jordan curve.

Proof. (6) $\Rightarrow$ (5). If $\Omega_{\lambda, \tau}$ is a Jordan curve, then $\rho^{+}(\theta)=\rho^{-}(\theta)$ for some $\theta \in \mathbb{R} / \mathbb{Z}$. Otherwise, $\rho^{+}(\theta)>\rho^{-}(\theta)$ for all $\theta$ and the two Jordan curves $\left\{\left(\theta, \rho^{+}(\theta)\right)\right\}$ and $\left\{\left(\theta, \rho^{-}(\theta)\right)\right\}$ would be disjoint and contained in the Jordan curve $\Omega_{\lambda, \tau}$ which is impossible.

(5) $\Rightarrow(4)$. If $\rho^{+}(\theta)=\rho^{-}(\theta)$ for some $\theta \in \mathbb{R} / \mathbb{Z}$, then the set $S=\left\{\theta \in \mathbb{R} / \mathbb{Z} \mid \rho^{+}(\theta)=\rho^{-}(\theta)\right\}$ is not empty, closed and $\mathbf{m}_{\ell}^{-1}(S)=S$, hence $S=\mathbb{R} / \mathbb{Z}$.

(4) $\Rightarrow$ (2). If $\rho^{+}$and $\rho^{-}$agree on $\mathbb{R} / \mathbb{Z}$, then $\Omega_{\lambda, \tau}$ is the graph of $\mu=\rho^{+}=\rho^{-}$.

$(2) \Rightarrow(3)$ (resp. $\left(2^{\prime}\right) \Rightarrow\left(3^{\prime}\right)$ ). If $\Omega_{\lambda, \tau} \subset \mathbb{R} / \mathbb{Z} \times \mathbb{R}$ is the graph of a $C^{0}$ (resp. $\mathcal{C}$ ) function $\mu: \mathbb{R} / \mathbb{Z} \rightarrow \mathbb{R}$, then $(\ell \theta, \lambda \mu(\theta)+\tau(\theta))=A_{\lambda, \tau}(\theta, \mu(\theta))$ belongs to the graph of $\mu$. Therefore, $\mu(\ell \theta)=\lambda \mu(\theta)+\tau(\theta)$ for all $\theta$.

(3) $\Rightarrow\left(3^{\prime}\right)$. Let us denote by $\pi: \mathbb{R} \rightarrow \mathbb{R} / \mathbb{Z}$ quotient map. If $\mu \circ \mathbf{m}_{\ell}-\lambda \mu=\tau$ has a $C^{0}$ solution $\mu: \mathbb{R} / \mathbb{Z} \rightarrow \mathbb{R}$, then $\mu \circ \pi: \mathbb{R} \rightarrow \mathbb{R}$ is a solution of $\tilde{\mu}(\ell s)-\lambda \tilde{\mu}(s)=\tilde{\tau}(s)$ where $\tilde{\tau}=\tau \circ \pi$. It is not difficult to check that this latter equation has a unique continuous solution given by

$$
\tilde{\mu}(s)=\tilde{\tau}(s / \ell)+\lambda \tilde{\tau}\left(s / \ell^{2}\right)+\lambda^{2} \tilde{\tau}\left(s / \ell^{3}\right)+\cdots,
$$

which is of the same class as $\tilde{\tau}$. Therefore, $\mu \circ \pi=\tilde{\mu}$ and $\mu$ are of class $\mathcal{C}$.

$\left(3^{\prime}\right) \Rightarrow\left(1^{\prime}\right)$ and $\left(2^{\prime}\right)$. If $\mu: \mathbb{R} / \mathbb{Z} \rightarrow \mathbb{R}$ is a $\mathcal{C}$ function such that $\mu \circ \mathbf{m}_{\ell}-\lambda \mu=\tau$, then $h \circ A_{\lambda, 0}=A_{\lambda, \tau} \circ h$ where $h(\theta, t)=(\theta, t+\mu(\theta))$. Hence $A_{\lambda, \tau}$ is $\mathcal{C}$-conjugate to $A_{\lambda, 0}$ and $\Omega_{\lambda, \tau}=h\left(\mathbb{R} / \mathbb{Z} \times\{0\}=\Omega_{\lambda, 0}\right)$ is the graph of $\mu: \mathbb{R} / \mathbb{Z} \rightarrow \mathbb{R}$.

Since $\left(1^{\prime}\right)$ trivially implies (1) and (1) implies (6), the proof of the proposition is complete.

\subsection{Infinite codimension}

Throughout this subsection we fix $\lambda \in(0,1)$. In Proposition 3.1 we showed that the global attractor $\Omega_{\lambda, \tau}$ of $A_{\lambda, \tau}$ is a Jordan curve if and only if the functional equation

$$
\tau=\mu \circ \mathbf{m}_{\ell}-\lambda \mu
$$


has a continuous solution $\mu: \mathbb{R} / \mathbb{Z} \rightarrow \mathbb{R}$. The aim of this subsection is to characterize the continuous functions $\tau: \mathbb{R} / \mathbb{Z} \rightarrow \mathbb{R}$ for which the functional equation (4) has a solution. With this purpose in mind, for each $k \in \mathbb{N}$, we introduce the linear functional

$$
\begin{aligned}
\mathcal{D}_{k}: L^{1}(\mathbb{R} / \mathbb{Z}, \mathbb{R}) & \rightarrow \mathbb{R}, \\
\psi & \mapsto \int_{\mathbb{R} / \mathbb{Z}} \psi(\theta) v_{k}(\theta) \mathrm{d} \theta
\end{aligned}
$$

where

$$
v_{k}(\theta)=\sum_{n \geqslant 0} \lambda^{n}\left(\mathrm{e}^{2 \pi \mathrm{i} \ell^{n} k \theta}+\mathrm{e}^{-2 \pi \mathrm{i} \ell^{n} k \theta}\right) .
$$

Lemma 3.2. Consider $\tau \in L^{1}(\mathbb{R} / \mathbb{Z}, \mathbb{R})$ and suppose that there exists $\mu \in L^{1}(\mathbb{R} / \mathbb{Z}, \mathbb{R})$ such that:

$$
\tau=\mu \circ \mathbf{m}_{\ell}-\lambda \mu \text {. }
$$

Then, $\mathcal{D}_{k}(\tau)=0$ for all $k \in \mathbb{N}$ such that $\ell \nmid k$.

Recall that for each $\phi \in L^{1}(\mathbb{R} / \mathbb{Z}, \mathbb{R})$ the $k$-th Fourier coefficient of $\phi$ is defined by

$$
\hat{\phi}(k)=\int_{\mathbb{R} / \mathbb{Z}} \phi(\theta) \mathrm{e}^{-2 \pi \mathrm{i} k \theta} \mathrm{d} \theta .
$$

For general background on Fourier series see [7].

Proof. From Eq. (4) it follows that

$$
\lambda^{n} \tau(\theta)+\lambda^{n-1} \tau(\ell \theta)+\cdots+\tau\left(\ell^{n} \theta\right)=\mu\left(\ell^{n+1} \theta\right)-\lambda^{n+1} \mu(\theta)
$$

for all $n \geqslant 1$. For all $k$ such that $\ell \nmid k$, computing the $\ell^{n} k$-th Fourier coefficient of the functions involved in the previous equation we obtain that:

$$
\lambda^{n} \hat{\tau}\left(\ell^{n} k\right)+\lambda^{n-1} \hat{\tau}^{n-1}\left(\ell^{n-1} k\right)+\cdots+\hat{\tau}(k)=-\lambda^{n+1} \hat{\mu}\left(\ell^{n} k\right) .
$$

In view of the fact that the Fourier coefficient of $\mu \in L^{1}(\mathbb{R} / \mathbb{Z}, \mathbb{R})$ are bounded, as $n \rightarrow \infty$ we have that $\lambda^{n+1} \hat{\mu}\left(\ell^{n} k\right) \rightarrow 0$ and, therefore,

$$
\sum_{n \geqslant 0} \lambda^{n} \hat{\tau}\left(\ell^{n} k\right)=0
$$

Now recall that the Fourier coefficients of a real valued function are even. In particular, $\hat{\tau}(k)=\hat{\tau}(-k)$ and Eq. (6) is equivalent to:

$$
\mathcal{D}_{k}(\tau)=\sum_{n \geqslant 0} \lambda^{n}\left(\hat{\tau}\left(\ell^{n} k\right)+\hat{\tau}\left(-\ell^{n} k\right)\right)=0 .
$$

Thus we have completed the proof of the lemma.

Theorem 2. Let $\tau: \mathbb{R} / \mathbb{Z} \rightarrow \mathbb{R}$ be a continuous function with absolutely convergent Fourier series. If

$$
\mathcal{D}_{k}(\tau)=0
$$

for all $k \in \mathbb{N}$ such that $\ell \nmid k$, then there exists a continuous function $\mu: \mathbb{R} / \mathbb{Z} \rightarrow \mathbb{R}$ satisfying the functional equation

$$
\tau=\mu \circ \mathbf{m}_{\ell}-\lambda \mu \text {. }
$$


Proof. We will obtain $\mu$ as a Fourier series with coefficients $b(\cdot)$ where:

$$
\begin{aligned}
b(0) & =\frac{\hat{\tau}(0)}{1-\lambda}, \\
b\left(\ell^{n} k\right) & =-\frac{1}{\lambda^{n+1}} \sum_{j=0}^{n} \lambda^{j} \hat{\tau}\left(\ell^{j} k\right)=\frac{1}{\lambda^{n+1}} \sum_{j=n+1}^{\infty} \lambda^{j} \hat{\tau}\left(\ell^{j} k\right)
\end{aligned}
$$

for $n \geqslant 0$ and $k \neq 0$ such that $\ell$ does not divide $k$. (Compare with Eq. (5).)

We claim that the Fourier series

$$
\sum_{q \in \mathbb{Z}} b(q) \mathrm{e}^{2 \pi \mathrm{i} q \theta}=b(0)+\sum_{\ell \nmid k} \sum_{n \geqslant 0} b\left(\ell^{n} k\right) \mathrm{e}^{2 \pi \mathrm{i} \ell^{n} k \theta}
$$

is absolutely convergent (i.e., $\sum_{q \in \mathbb{Z}}|b(q)|<\infty$ ). In fact, from Eq. (8):

$$
\sum_{q \in \mathbb{Z}}|b(q)|=|b(0)|+\sum_{\ell \nmid k} \sum_{n \geqslant 0}\left|b\left(\ell^{n} k\right)\right| \leqslant|b(0)|+\sum_{\ell \nmid k} \sum_{n \geqslant 0} \frac{1}{\lambda^{n+1}} \sum_{j \geqslant n+1} \lambda^{j}\left|\hat{\tau}\left(\ell^{j} k\right)\right| .
$$

Since

$$
\sum_{n \geqslant 0} \frac{1}{\lambda^{n+1}} \sum_{j \geqslant n+1} \lambda^{j}\left|\hat{\tau}\left(\ell^{j} k\right)\right|=\sum_{n \geqslant 0} \sum_{j=0}^{n} \lambda^{j}\left|\hat{\tau}\left(\ell^{n+1} k\right)\right|=\sum_{n \geqslant 0} \frac{1-\lambda^{n+1}}{1-\lambda}\left|\cdot \hat{\tau}\left(\ell^{n+1} k\right)\right| \leqslant \frac{1}{1-\lambda} \sum_{n \geqslant 0}\left|\hat{\tau}\left(\ell^{n+1} k\right)\right|,
$$

it follows that

$$
\sum_{q \in \mathbb{Z}}|b(q)| \leqslant|\hat{\tau}(0)| \frac{1}{1-\lambda}+\frac{1}{1-\lambda} \sum_{\ell \nmid k} \sum_{n \geqslant 0}\left|\hat{\tau}\left(\ell^{n+1} k\right)\right|=\frac{1}{1-\lambda} \sum_{q \in \mathbb{Z}}|\hat{\tau}(q)|<\infty .
$$

Thus the series $\sum_{q \in \mathbb{Z}} b(q) \mathrm{e}^{2 \pi \mathrm{i} q \theta}$ uniformly converges to a continuous function $\mu: \mathbb{R} / \mathbb{Z} \rightarrow \mathbb{R}$. which is a solution of $\tau=\mu \circ \mathbf{m}_{\ell}-\lambda \mu$ since an easy computation shows that the Fourier coefficients of $\tau$ and $\mu \circ \mathbf{m}_{\ell}-\lambda \mu$ agree (cf. Lemma 3.2).

Definition 3.3. Let $\tau: \mathbb{R} / \mathbb{Z} \rightarrow \mathbb{R}$ be a continuous function with absolutely convergent Fourier series. Define the canonical representative of $\tau$ by the continuous function $\tau_{c}: \mathbb{R} / \mathbb{Z} \rightarrow \mathbb{R}$ given by the Fourier series $\tau_{c}(\theta)=$ $\sum_{\ell \nmid k} \mathcal{D}_{k}(\tau) \mathrm{e}^{2 \pi \mathrm{i} k \theta}$.

An immediate consequence of the theorem above is the following corollary:

Corollary 3.4. Let $\tau: \mathbb{R} / \mathbb{Z} \rightarrow \mathbb{R}$ be a continuous function with absolutely convergent Fourier series. If $\tau_{c}$ is the canonical representative of $\tau$, then $\tau$ is cohomologous to $\tau_{c}$. That is, there exists a continuous function $\mu: \mathbb{R} / \mathbb{Z} \rightarrow \mathbb{R}$ such that $\tau=\tau_{c}+\mu \circ m_{\ell}-\lambda \mu$.

Proof. Note that for every $k$ which is not divided by $\ell$ it holds that $\mathcal{D}_{k}\left(\tau-\tau_{c}\right)=0$. Therefore we may apply the previous theorem to $\tau-\tau_{c}$ and obtain the corollary.

\section{Annular attractors}

Our next result contains the sufficient condition for $\Omega_{\lambda, \tau}$ to be a closed topological annulus which will be used in the proof of Theorem 1. More precisely, below we prove that if the image of the upper boundary of $\Omega_{\lambda, \tau}$ lies higher in $\mathbb{R} / \mathbb{Z} \times \mathbb{R}$ than the image of the lower boundary, then $\Omega_{\lambda, \tau}$ is an annulus: 
Lemma 4.1. If $\Omega_{\lambda, \tau}$ is not a Jordan curve and

$$
\min \left\{\lambda \rho^{+}\left(\theta^{\prime}\right)+\tau\left(\theta^{\prime}\right) \mid \theta^{\prime} \in \mathbf{m}_{\ell}^{-1}(\theta)\right\} \geqslant \max \left\{\lambda \rho^{-}\left(\theta^{\prime}\right)-\tau\left(\theta^{\prime}\right) \mid \theta^{\prime} \in \mathbf{m}_{\ell}^{-1}(\theta)\right\}
$$

for all $\theta \in \mathbb{R} / \mathbb{Z}$, then $\Omega_{\lambda, \tau}$ is a closed topological annulus. Moreover, if $\ell=2$ and $\Omega_{\lambda, \tau}$ is a closed topological annulus, then inequality (9) holds.

Remark 4.2. For all $\ell \geqslant 2$ and provided that $\Omega_{\lambda, \tau}$ is not a Jordan curve, a necessary and sufficient condition for $\Omega_{\lambda, \tau}$ to be homeomorphic to a closed annulus is that for all $\theta \in \mathbb{R} / \mathbb{Z}$ :

$$
I(\theta)=\bigcup_{\theta^{\prime} \in \mathbf{m}_{\ell}^{-1}(\theta)} A_{\lambda, \tau}\left(I\left(\theta^{\prime}\right)\right)
$$

where $I(\theta)=\{\theta\} \times\left[\rho^{-}(\theta), \rho^{+}(\theta)\right]$.

Proof. Let $\mathcal{A}=\left\{(\theta, t) \mid \rho^{-}(\theta) \leqslant t \leqslant \rho^{+}(\theta)\right\}$ and note that $A_{\lambda, \tau}(\mathcal{A}) \subset \mathcal{A}$. We claim that from (9) it follows $A_{\lambda, \tau}(\mathcal{A})=\mathcal{A}$ and therefore $\Omega_{\lambda, \tau}=\mathcal{A}$. Otherwise there would exist $t_{0} \in\left(\rho^{-}\left(\theta_{0}\right), \rho^{+}\left(\theta_{0}\right)\right)$ such that $\left(\theta_{0}, t_{0}\right) \notin$ $A_{\lambda, \tau}(\mathcal{A})$. Consider $\theta_{ \pm}^{\prime} \in \mathbf{m}_{\ell}^{-1}\left(\theta_{0}\right)$ such that $\rho^{ \pm}\left(\theta_{0}\right)=\lambda \rho^{ \pm}\left(\theta_{ \pm}^{\prime}\right)+\tau\left(\theta_{ \pm}^{\prime}\right)$. Since $\left(\theta_{0}, t_{0}\right) \notin A_{\lambda, \tau}(\mathcal{A})$, the image of $\mathcal{A} \cap\left(\left\{\theta_{+}^{\prime}\right\} \times \mathbb{R}\right)$ should be above $t_{0}$ and the image of $\mathcal{A} \cap\left(\left\{\theta_{-}^{\prime}\right\} \times \mathbb{R}\right)$ should be below $t_{0}$. Hence, we would have:

$$
\lambda \rho^{+}\left(\theta_{-}^{\prime}\right)+\tau\left(\theta_{-}^{\prime}\right)<t_{0}<\lambda \rho^{-}\left(\theta_{+}^{\prime}\right)+\tau\left(\theta_{+}^{\prime}\right)
$$

which contradicts (9). Therefore $\mathcal{A}=\Omega_{\lambda, \tau}$. By Proposition 3.1, if $\Omega_{\lambda, \tau}$ is not a Jordan curve, then $\rho^{+}>\rho^{-}$on $\mathbb{R} / \mathbb{Z}$. Hence $\Omega_{\lambda, \tau}=\mathcal{A}$ is a closed topological annulus.

For $\ell=2$, if (9) does not hold for some $\theta_{0}$, then the two intervals, $A_{\lambda, \tau}\left(\mathcal{A} \cap\left(\left\{\theta_{0} / 2\right\} \times \mathbb{R}\right)\right)$ and $A_{\lambda, \tau}(\mathcal{A} \cap$ $\left.\left(\left\{\theta_{0} / 2+1 / 2\right\} \times \mathbb{R}\right)\right)$ are disjoint and their union is $A_{\lambda, \tau}(\mathcal{A}) \cap\left(\left\{\theta_{0}\right\} \times \mathbb{R}\right)$. Therefore, $\Omega_{\lambda, \tau} \subset A_{\lambda, \tau}(\mathcal{A})$ is not an annulus.

To show that if $\Omega_{\lambda, \tau}$ is a topological annulus then $\lambda>1 / \ell$, we will need the following result.

Lemma 4.3. Assume that $\lambda \leqslant 1 / \ell$. Let $\tau: \mathbb{R} / \mathbb{Z} \rightarrow \mathbb{R}$ be a continuous function. If $\rho^{+}-\rho^{-}$is a constant function, then $\Omega_{\lambda, \tau}$ is not an annulus.

Proof. Suppose that $\lambda \leqslant 1 / \ell$ and $\rho^{+}-\rho^{-}$is the constant function $C$ for some $C>0$. We proceed by contradiction. If $\Omega_{\lambda, \tau}$ is an annulus, then

$$
\left[\rho^{-}(\theta), \rho^{+}(\theta)\right]=\bigcup_{\theta^{\prime} \in \mathbf{m}_{\ell}^{-1}(\theta)} J\left(\theta^{\prime}\right)
$$

where $J\left(\theta^{\prime}\right)=\left[\lambda \rho^{-}\left(\theta^{\prime}\right)+\tau\left(\theta^{\prime}\right), \lambda \rho^{+}\left(\theta^{\prime}\right)+\tau\left(\theta^{\prime}\right)\right]$. Since the length of $\left[\rho^{-}(\theta), \rho^{+}(\theta)\right]$ is $C$ and the length of each of the $\ell$ intervals $J\left(\theta^{\prime}\right)$ is $\lambda C$, it follows that $\ell \lambda C \geqslant C$ and therefore $\lambda=1 / \ell$. Moreover, the interior of the intervals $J\left(\theta^{\prime}\right)$ must be pairwise disjoint, which is impossible since the image under $A_{\lambda, \tau}$ of the loop $\left\{\left(\theta,\left(\rho^{+}(\theta)+\right.\right.\right.$ $\left.\left.\left.\rho^{-}(\theta)\right) / 2\right) \mid \theta \in \mathbb{R} / \mathbb{Z}\right\}$ must have self-intersections.

Proposition 4.4. If $\Omega_{\lambda, \tau}$ is a closed topological annulus, then $\lambda>1 / \ell$.

Proof. Suppose that $\lambda \leqslant 1 / \ell$. Let $C$ be the maximum of $\rho^{+}-\rho^{-}$and let $E \subset \mathbb{R} / \mathbb{Z}$ be the set formed by the arguments $\theta$ such that $C=\left(\rho^{+}-\rho^{-}\right)(\theta)$. From the previous lemma we may assume that $E \neq \mathbb{R} / \mathbb{Z}$. Hence, there exists $\theta_{0} \notin E$ such that $\theta=\ell \theta_{0} \in E$. It follows that the length of

$$
S=\bigcup_{\theta^{\prime} \in \mathbf{m}_{\ell}^{-1}(\theta)}\left[\lambda \rho^{-}\left(\theta^{\prime}\right)+\tau\left(\theta^{\prime}\right), \lambda \rho^{+}\left(\theta^{\prime}\right)+\tau\left(\theta^{\prime}\right)\right]
$$


is strictly less than $C$. Therefore, $\left[\rho^{-}(\theta), \rho^{+}(\theta)\right] \neq S$ and by Remark 4.2 we conclude that $\Omega_{\lambda, \tau}$ is not an annulus.

\subsection{Annular attractors when $\tau$ is not cohomologous to 0}

The aim of this subsection is to prove (2) of Theorem 1 under some extra assumptions on $\tau$.

Definition 4.5. We say that $\tau: \mathbb{R} / \mathbb{Z} \rightarrow \mathbb{R}$ is cohomologous to 0 or a coboundary if $\tau=\mu \circ \mathbf{m}_{\ell}-\mu$ for some continuous $\mu: \mathbb{R} / \mathbb{Z} \rightarrow \mathbb{R}$.

We will show that Theorem 1 (2) holds if $\tau$ is not cohomologous to 0 and $\int_{\mathbb{R} / \mathbb{Z}} \tau(\theta) \mathrm{d} \theta=0$. Under these stronger assumptions we can weaken the Lipschitz class hypothesis for $\tau$ :

Proposition 4.6. Let $\tau: \mathbb{R} / \mathbb{Z} \rightarrow \mathbb{R}$ be a Hölder function which is not cohomologous to 0 and such that $\int_{\mathbb{R} / \mathbb{Z}} \tau(\theta) \mathrm{d} \theta=0$. Then there exists $\lambda_{0}<1$ such that $\Omega_{\lambda, \tau}$ is a closed topological annulus for all $\lambda \in\left[\lambda_{0}, 1\right)$.

The proof relies on finding appropriate periodic orbits under the multiplication by $\ell$ map $\mathbf{m}_{\ell}: \mathbb{R} / \mathbb{Z} \rightarrow \mathbb{R} / \mathbb{Z}$.

To simplify notation we let $\int \tau=\int_{\mathbb{R} / \mathbb{Z}} \tau(\theta) \mathrm{d} \theta=0$ since we will only consider integrals with respect to the Lebesgue measure on $\mathbb{R} / \mathbb{Z}$.

Lemma 4.7. If $\tau: \mathbb{R} / \mathbb{Z} \rightarrow \mathbb{R}$ is a Hölder function which is not cohomologous to 0 and such that $\int \tau=0$, then there exist periodic points $\theta_{ \pm}$of period $p_{ \pm}$such that:

$$
\begin{gathered}
\tau\left(\theta_{+}\right)+\cdots+\tau\left(\ell^{p_{+}} \theta_{+}\right)>0, \\
\tau\left(\theta_{-}\right)+\cdots+\tau\left(\ell^{p_{-}} \theta_{-}\right)<0 .
\end{gathered}
$$

We employ the ideas contained in the proof of Theorem 9 in [2].

Proof. By considering $-\tau$ instead of $\tau$ it is sufficient to find $\theta_{+}$. For $\bar{\theta}=\left(\theta_{0}, \theta_{1}, \theta_{2}, \ldots\right) \in \mathcal{S}$ we let

$$
S_{n} \bar{\theta}=\tau\left(\theta_{1}\right)+\cdots+\tau\left(\theta_{n}\right) .
$$

We proceed by contradiction and suppose that for all period $n$ points $\bar{\theta}$ we have that $S_{n} \bar{\theta} \leqslant 0$.

We claim that

$$
\left\{S_{n} \bar{\theta} \mid \bar{\theta} \in \mathcal{S}, n \in \mathbb{N}\right\}
$$

is bounded above. In fact, for any $\bar{\theta} \in \mathcal{S}$ there exists a period $n$ point $\bar{\theta}^{\prime}=\left(\theta_{0}^{\prime}, \theta_{1}^{\prime}, \ldots\right) \in \mathcal{S}$ such that

$$
\operatorname{dist}_{\mathbb{R} / \mathbb{Z}}\left(\theta_{n}^{\prime}, \theta_{n}\right) \leqslant \frac{1}{\ell^{n-1}} .
$$

Therefore,

$$
S_{n}(\bar{\theta}) \leqslant S_{n}(\bar{\theta})-S_{n}\left(\bar{\theta}^{\prime}\right) \leqslant C \cdot\left(1+\cdots+\ell^{-(n-1) \alpha}\right) \leqslant C \cdot \frac{\ell^{\alpha}}{\ell^{\alpha}-1}
$$

where $C>0$ is an $\alpha$-Hölder constant for $\tau$.

Now $\mu: \mathbb{R} / \mathbb{Z} \rightarrow \mathbb{R}$ defined by

$$
\mu\left(\theta_{0}\right)=\sup \left\{S_{n} \bar{\theta} \mid \bar{\theta}=\left(\theta_{0}, \beta_{1}, \beta_{2}, \ldots\right) \in \mathcal{S}\right\}
$$

is an $\alpha$-Hölder function. In fact, for any $\theta_{0}, \theta_{0}^{\prime} \in \mathbb{R} / \mathbb{Z}$ and $\varepsilon>0$ let $\bar{\theta}=\left(\theta_{0}, \theta_{1}, \ldots\right)$ be such that $\mu\left(\theta_{0}\right)<S_{n} \bar{\theta}+\varepsilon$. Then there exists $\bar{\theta}^{\prime}=\left(\theta_{0}^{\prime}, \ldots\right) \in \mathcal{S}$ such that $\operatorname{dist}_{\mathbb{R} / \mathbb{Z}}\left(\theta_{k}, \theta_{k}^{\prime}\right)=\ell^{-k} \operatorname{dist}_{\mathbb{R} / \mathbb{Z}}\left(\theta_{0}, \theta_{0}^{\prime}\right)$. Therefore, 


$$
\begin{aligned}
\mu\left(\theta_{0}^{\prime}\right) \geqslant S_{n}\left(\bar{\theta}^{\prime}\right) & =S_{n}\left(\bar{\theta}^{\prime}\right)-S_{n}(\bar{\theta})+S_{n}(\bar{\theta}) \geqslant S_{n}(\bar{\theta})-C \frac{1}{\ell^{\alpha}-1} \operatorname{dist}_{\mathbb{R} / \mathbb{Z}}\left(\theta_{0}, \theta_{0}^{\prime}\right)^{\alpha} \\
& \geqslant \mu\left(\theta_{0}\right)-\varepsilon-C \frac{1}{\ell^{\alpha}-1} \operatorname{dist}_{\mathbb{R} / \mathbb{Z}}\left(\theta_{0}, \theta_{0}^{\prime}\right)^{\alpha} .
\end{aligned}
$$

It follows that $\mu$ is $\alpha$-Hölder, in particular continuous.

From the definition of $\mu$ we conclude that

$$
\mu(\ell \theta) \geqslant \mu(\theta)+\tau(\theta) .
$$

Therefore $\mu \circ \mathbf{m}_{\ell}-\mu-\tau$ is a non-negative function whose integral over $\mathbb{R} / \mathbb{Z}$ is zero. It follows that $\tau=\mu \circ \mathbf{m}_{\ell}-\mu$ which contradicts our assumption that $\tau$ is not cohomologous to 0 .

Proof of Proposition 4.6. We will show that $\rho^{+} \rightarrow+\infty$ (resp. $\left.\rho^{-} \rightarrow-\infty\right)$ as $\lambda \rightarrow 1$. In particular for $\lambda$ sufficiently close to 1 we have that $\rho^{+}>\lambda^{-1}\|\tau\|_{\infty}$ (resp. $\rho^{-}<-\lambda^{-1}\|\tau\|_{\infty}$ ), which in view of Lemma 4.1 implies that $\Omega_{\lambda, \tau}$ is an annulus.

Let $\theta_{+}$be a period $p=p_{+}$periodic point as in the previous lemma and let $c=\tau\left(\theta_{+}\right)+\cdots+\tau\left(\ell^{p-1} \theta_{+}\right)>0$. Consider $\lambda_{1}<1$ and a neighborhood $U \subset \mathbb{R} / \mathbb{Z}$ of $\mathcal{O}=\left\{\theta_{+}, \ldots, \ell^{p-1} \theta_{+}\right\}$such that:

(a) There exists a neighborhood $V \subset U$ of $\mathcal{O}$ such that $\mathbf{m}_{\ell}: V \rightarrow U$ is a bijection.

(b) For all $\lambda>\lambda_{1}$, if $\left\{\theta, \ell \theta, \ldots, \ell^{p-1} \theta\right\} \subset U$, then

$$
\tau(\theta)+\lambda \tau(\ell \theta)+\cdots+\lambda^{p-1} \tau\left(\ell^{p-1} \theta\right)>\frac{c}{2} .
$$

Let $n_{0}$ be such that $\mathbf{m}_{\ell}^{n_{0}}(U)=\mathbb{R} / \mathbb{Z}$. Then for all $\theta_{0} \in \mathbb{R} / \mathbb{Z}$ there exists $\bar{\theta}=\left(\theta_{0}, \ldots\right) \in \mathcal{S}$ such that $\theta_{n} \in U$ for all $n \geqslant n_{0}$. Hence for all $\lambda>\lambda_{1}$,

$$
\begin{aligned}
\rho^{+}\left(\theta_{0}\right) & \geqslant \sum_{k \geqslant 1} \lambda^{k-1} \tau\left(\theta_{k}\right)=\sum_{k \geqslant 1}^{n_{0}-1} \lambda^{k-1} \tau\left(\theta_{k}\right)+\sum_{k \geqslant n_{0}} \lambda^{k-1} \tau\left(\theta_{k}\right) \\
& \geqslant-\left(1+\cdots+\lambda^{n_{0}-1}\right)\|\tau\|_{\infty}+\lambda^{n_{0}-1}\left(1+\lambda^{p}+\lambda^{2 p}+\cdots\right) \frac{c}{2} \\
& \geqslant-n_{0}\|\tau\|_{\infty}+\frac{c \lambda^{n_{0}-1}}{2\left(1-\lambda^{p}\right)} .
\end{aligned}
$$

Remark 4.8. Provided that $\tau: \mathbb{R} / \mathbb{Z} \rightarrow \mathbb{R}$ is Hölder, $\int \tau=0$ and $\tau$ is not cohomologous to zero, it follows from the previous proof that for $\lambda \in\left[\lambda_{0}, 1\right)$ the inequality (9) holds strictly, that is:

$$
\min \left\{\lambda \rho^{+}\left(\theta^{\prime}\right)+\tau\left(\theta^{\prime}\right) \mid \theta^{\prime} \in \mathbf{m}_{\ell}^{-1}(\theta)\right\}>\max \left\{\lambda \rho^{-}\left(\theta^{\prime}\right)+\tau\left(\theta^{\prime}\right) \mid \theta^{\prime} \in \mathbf{m}_{\ell}^{-1}(\theta)\right\} .
$$

\section{Cohomological operators}

In this section we will study the linear operators

$$
\mathcal{L}_{\lambda} \mu=\mu \circ \mathbf{m}_{\ell}-\lambda \mu
$$

acting on Lipschitz functions $\mu: \mathbb{R} / \mathbb{Z} \rightarrow \mathbb{R}$ where $\lambda \in(0,1]$. On one hand these linear operators are related to conjugacy classes of affine maps $A_{\lambda, \tau}$ (see Lemma 5.8). On the other hand, in view of Propositions 3.1 and 4.6, these operators are also related to topological properties of the attractor $\Omega_{\lambda, \tau}$. At the end of this section we will apply the properties of $\mathcal{L}_{\lambda}$ to prove a stronger version of Theorem 1 announced in Subsection 1.3. 


\subsection{Basic properties}

Definition 5.1. Consider a continuous function $\tau: \mathbb{R} / \mathbb{Z} \rightarrow \mathbb{R}$. Given $\lambda \in(0,1]$ we define the multiplicity mult $\tau(\lambda)$ as follows: $\operatorname{mult}_{\tau}(\lambda)=\sup \left\{n \geqslant 0 \mid\right.$ there is a continuous function $\mu: \mathbb{R} / \mathbb{Z} \rightarrow \mathbb{R}$ such that $\left.\mathcal{L}_{\lambda}^{n} \mu=\tau\right\}$.

We will show that $\mathcal{J}_{\tau}^{\prime}=\left\{\lambda \in(0,1] \mid \operatorname{mult}_{\tau}(\lambda)>0\right\}$ is finite (counting multiplicities) provided that $\tau$ is a nonconstant Lipschitz function (cf. Main Lemma). In particular, mult $_{\tau}(\lambda)$ is finite for each $\lambda \in(0,1]$.

Although our main interest is on maps $A_{\lambda, \tau}$ where $\tau: \mathbb{R} / \mathbb{Z} \rightarrow \mathbb{R}$ is Lipschitz, the basic properties of the operators $\mathcal{L}_{\lambda}$ acting on any class are summarized in the following lemma.

Lemma 5.2. For $\lambda \in(0,1]$, let $\mathcal{L}_{\lambda}: \mathcal{C} \rightarrow \mathcal{C}$ be the linear operator defined above acting on the class $\mathcal{C}$ of maps from $\mathbb{R} / \mathbb{Z}$ to $\mathbb{R}$ where $\mathcal{C}$ is the class of Lipschitz or $C^{r}$ maps for some $r \in[0, \infty] \cup\{\omega\}$. Then:

(1) $\mathcal{L}_{\lambda_{1}} \circ \mathcal{L}_{\lambda_{2}}=\mathcal{L}_{\lambda_{2}} \circ \mathcal{L}_{\lambda_{1}}$ for all $0<\lambda_{1}, \lambda_{2} \leqslant 1$.

(2) If $\mathcal{L}_{\lambda_{1}} \mu_{1}=\tau$, then mult $\mu_{1}(\lambda)=$ mult $_{\tau}(\lambda)$ for all $\lambda \neq \lambda_{1}$ and mult $_{\mu_{1}}\left(\lambda_{1}\right)=$ mult $_{\tau}\left(\lambda_{1}\right)-1$.

(3) $\operatorname{ker} \mathcal{L}_{1}=\{$ constant maps $\}$ and $\operatorname{ker} \mathcal{L}_{\lambda}=\{0\}$ for all $\lambda \in(0,1)$.

(4) If $\mathcal{L}_{\lambda} \mu=\tau$ and $\int \tau=0$ for some $\lambda \in(0,1)$, then $\int \mu=0$.

(5) If $\tau: \mathbb{R} / \mathbb{Z} \rightarrow \mathbb{R}$ is of class $\mathcal{C}$ and $\mu: \mathbb{R} / \mathbb{Z} \rightarrow \mathbb{R}$ is a continuous function such that $\mu \circ \mathbf{m}_{\ell}-\lambda \mu=\tau$, then $\mu$ is of class $\mathcal{C}$.

(6) If $\tau \in \operatorname{Image} \mathcal{L}_{1}$, then $\int \tau=0$.

(7) $\mathcal{L}_{\lambda}(\{$ constant maps $\})=\{$ constant maps $\}$ for all $\lambda \in(0,1)$.

Proof. Statement (1) is a straightforward computation and statements (4) and (6) are an immediate consequence of the fact that the Lebesgue measure in the circle is $\mathbf{m}_{\ell}$-invariant (i.e., $\int \tau \circ \mathbf{m}_{\ell}=\int \tau$ for all continuous $\tau: \mathbb{R} / \mathbb{Z} \rightarrow \mathbb{R})$.

For (2), suppose that $\mathcal{L}_{\lambda_{1}} \mu_{1}=\tau$ and just note that if, for some $\lambda \neq \lambda_{1}$, there exists $\mu$ such that $\mathcal{L}_{\lambda} \mu=\tau$, then $\mathcal{L}_{\lambda}\left(\mu-\mu_{1}\right) /\left(\lambda-\lambda_{1}\right)=\mu_{1}$.

(3) If $\mu(\ell \theta)-\lambda \mu(\theta)=0$, then $\mu\left(\ell^{n} \theta\right)=\lambda^{n} \mu(\theta)$ for all $\theta \in \mathbb{R} / \mathbb{Z}$ and all $n \geqslant 0$. Let $\theta_{0}$ be such that $\left\{\ell^{n} \theta_{0}\right\}_{n} \geqslant 0$ is dense in $\mathbb{R} / \mathbb{Z}$. Hence, for all $\theta \in \mathbb{R} / \mathbb{Z}, \mu(\theta)=0$ when $\lambda<1$ and $\mu(\theta)=\mu\left(\theta_{0}\right)$ when $\lambda=1$.

(5) As in the proof of Proposition 3.1 we pass to the universal cover $\pi: \mathbb{R} \rightarrow \mathbb{R} / \mathbb{Z}$. That is, if $\mu \circ \mathbf{m}_{\ell}-\lambda \mu=\tau$ then $\tilde{\mu}(\ell s)-\lambda \tilde{\mu}(s)=\tilde{\tau}(s)$ where $\tilde{\mu}=\mu \circ \pi$ and $\tilde{\tau}=\tau \circ \pi$. It follows that $\tilde{\mu}(s)=\tilde{\tau}\left(\ell^{-1} s\right)+\lambda \tilde{\tau}\left(\ell^{-2} s\right)+\cdots$ is a class $\mathcal{C}$ map from $\mathbb{R}$ to $\mathbb{R}$ and therefore $\mu: \mathbb{R} / \mathbb{Z} \rightarrow \mathbb{R}$ is also of class $\mathcal{C}$.

Since the image under $\mathcal{L}_{\lambda}$ of the constant function $\tau$ equal to 1 is the constant function $\mu$ equal to $1-\lambda$, statement (7) follows.

Remark 5.3. From (1) and (2) it follows that there exists a continuous $\mu$ such that $\mathcal{L}_{\lambda_{1}} \circ \cdots \circ \mathcal{L}_{\lambda_{m}} \mu=\tau$ if and only if $\lambda_{1}, \ldots, \lambda_{m}$ is a collection of elements of $\mathcal{J}_{\tau}^{\prime}$, maybe with repetitions, but such that the number of occurrences of $\lambda \in \mathcal{J}_{\tau}^{\prime}$ is not greater than its multiplicity. Moreover, (7) implies that $\mu$ is uniquely determined by $\tau$ and $\lambda_{1}, \ldots, \lambda_{m}$ up to an additive constant.

Lemma 5.4. If $\mu: \mathbb{R} / \mathbb{Z} \rightarrow \mathbb{R}$ is Lipschitz, then

$$
\left\|\mathcal{L}_{\lambda} \mu\right\|_{L} \geqslant(\ell-\lambda)\|\mu\|_{L}
$$

for all $\lambda \in(0,1]$.

Proof. Let $\mu$ be Lipschitz and let $\tau=\mathcal{L}_{\lambda} \mu$. Consider $\bar{\theta}=\left(\theta_{k}\right) \in \mathcal{S}$ and observe that

$$
\mu\left(\theta_{0}\right)=\tau\left(\theta_{1}\right)+\lambda \tau\left(\theta_{2}\right)+\ldots+\lambda^{n} \tau\left(\theta_{n+1}\right)+\lambda^{n+1} \mu\left(\theta_{n+1}\right) .
$$


For $\theta_{0}^{\prime} \in \mathbb{R} / \mathbb{Z}$ let $\bar{\theta}^{\prime}=\left(\theta_{k}^{\prime}\right)$ be such that

$$
\operatorname{dist}_{\mathbb{R} / \mathbb{Z}}\left(\theta_{k}, \theta_{k}^{\prime}\right)=\frac{\operatorname{dist}_{\mathbb{R} / \mathbb{Z}}\left(\theta_{0}, \theta_{0}^{\prime}\right)}{\ell^{k}} .
$$

It follows that for all $n \geqslant 0$ :

$$
\begin{aligned}
\left|\mu\left(\theta_{0}\right)-\mu\left(\theta_{0}^{\prime}\right)\right| & \leqslant \operatorname{dist}_{\mathbb{R} / \mathbb{Z}}\left(\theta_{0}, \theta_{0}^{\prime}\right)\|\tau\|_{L}\left(\frac{1}{\ell}+\frac{\lambda}{\ell^{2}}+\ldots+\frac{\lambda^{n}}{\ell^{n+1}}\right)+\lambda^{n+1}\left|\mu\left(\theta_{n+1}\right)-\mu\left(\theta_{n+1}^{\prime}\right)\right| \\
& \leqslant \operatorname{dist}_{\mathbb{R} / \mathbb{Z}}\left(\theta_{0}, \theta_{0}^{\prime}\right)\|\tau\|_{L} \frac{1}{\ell-\lambda}+\lambda^{n+1}\left|\mu\left(\theta_{n+1}\right)-\mu\left(\theta_{n+1}^{\prime}\right)\right| .
\end{aligned}
$$

Therefore,

$$
\left|\mu\left(\theta_{0}\right)-\mu\left(\theta_{0}^{\prime}\right)\right| \leqslant\|\tau\|_{L} \frac{1}{\ell-\lambda} \operatorname{dist}_{\mathbb{R} / \mathbb{Z}}\left(\theta_{0}, \theta_{0}^{\prime}\right)
$$

and the lemma follows.

Lemma 5.5. Let $\tau: \mathbb{R} / \mathbb{Z} \rightarrow \mathbb{R}$ be a Lipschitz map such that $\int \tau=0$. The set

$$
\mathcal{J}_{\tau}^{\prime}=\left\{\lambda \in(0,1] \mid \operatorname{mult}_{\tau}(\lambda)>0\right\}
$$

is closed in $(0,1]$.

Note that from Proposition 3.1 it follows that

$$
\mathcal{J}_{\tau}^{\prime} \cap(0,1)=\mathcal{J}_{\tau}=\left\{\lambda \in(0,1) \mid \Omega_{\lambda, \tau} \text { is a Jordan curve }\right\} .
$$

Proof. Suppose that there exists $\lambda_{n} \rightarrow \lambda \in(0,1]$ and $\mu_{n}: \mathbb{R} / \mathbb{Z} \rightarrow \mathbb{R}$ such that $\mu_{n} \circ \mathbf{m}_{\ell}-\lambda_{n} \mu=\tau$. It follows that $\int \mu_{n}=0$ and $\left\|\mu_{n}\right\|_{L} \leqslant\|\tau\|_{L}$ for all $n$. Therefore, $\left\{\mu_{n}\right\}$ is an equicontinuous and uniformly bounded family. Hence, by passing to a subsequence, we may assume that $\left\{\mu_{n}\right\}$ converges to some continuous function $\mu$ which necessarily satisfies the equation $\mu \circ \mathbf{m}_{\ell}-\lambda \mu=\tau$, that is, $\lambda \in \mathcal{J}_{\tau}^{\prime}$.

\subsection{Finiteness results}

We now show that a Lipschitz function $\tau$ which is not constant is not "infinitely" cohomologous to 0 or equivalently that $\lambda=1$ has finite multiplicity (see Definition 5.1).

Lemma 5.6. Let $\tau: \mathbb{R} / \mathbb{Z} \rightarrow \mathbb{R}$ be a non-constant Lipschitz function such that $\int \tau=0$. Then there exists $m \geqslant 0$ and a Lipschitz function $\mu: \mathbb{R} / \mathbb{Z} \rightarrow \mathbb{R}$ such that $\mathcal{L}_{1}^{m} \mu=\tau$ and $\int \mu=0$ but $\mathcal{L}_{1} \rho \neq \mu$ for all Lipschitz functions $\rho$.

Proof. Suppose that for $0 \leqslant n \leqslant m$ there exist $\mu_{n}: \mathbb{R} / \mathbb{Z} \rightarrow \mathbb{R}$ such that

$$
\mathcal{L}_{1}^{n} \mu_{n}=\mu_{0}=\tau \text {. }
$$

By Lemma 5.2, maybe after adding a constant to $\mu_{m}$, we may assume that for $0 \leqslant n \leqslant m$ we have $\int \mu_{n}=0$. It follows that $\mathcal{L}_{1} \mu_{n}=\mu_{n-1}$. Under the assumption that $\tau=\mu_{0}$ is not identically 0 we will exhibit an upper bound for $m$ in terms of the Fourier coefficients of $\tau$.

The Fourier coefficients $\hat{\mu}_{n}(k)$ are uniformly bounded. In fact, by Lemma 5.4, $\left\|\mu_{n}\right\|_{L} \leqslant\|\tau\|_{L}$ and therefore

$$
\left|\hat{\mu}_{n}(k)\right| \leqslant \frac{\|\tau\|_{L}}{4 k}
$$

for all $0 \neq k \in \mathbb{Z}$ and $0 \leqslant n \leqslant m$. 
Since $\mu_{0}$ is not identically 0 , there exists $k \in \mathbb{Z}$ such that $\ell \nmid k$ and $p \geqslant 0$ such that:

$$
\begin{aligned}
& \hat{\mu}_{0}\left(\ell^{j} k\right)=0 \quad \text { for } 0 \leqslant j<p, \\
& \hat{\mu}_{0}\left(\ell^{p} k\right) \neq 0 .
\end{aligned}
$$

Taking the $\ell^{j} k$-th Fourier coefficient to $\mu_{n} \circ \mathbf{m}_{\ell}-\mu_{n}=\mu_{n-1}$ :

$$
\begin{aligned}
& \hat{\mu}_{n}\left(\ell^{j-1} k\right)-\hat{\mu}_{n}\left(\ell^{j} k\right)=\hat{\mu}_{n-1}\left(\ell^{j} k\right) \quad \text { for } j \geqslant 1, \\
& -\hat{\mu}_{n}(k)=\hat{\mu}_{n-1}(k),
\end{aligned}
$$

for $1 \leqslant n \leqslant m$.

By induction in $p \geqslant 0$, it is easy to deduce from (11) and (12) that if $\hat{\mu}_{0}\left(\ell^{j} k\right)=0$ for $0 \leqslant j<p$, then:

$$
\begin{aligned}
& \hat{\mu}_{n}\left(\ell^{j} k\right)=0 \quad \text { for } 0 \leqslant j<p, \\
& -\hat{\mu}_{n}\left(\ell^{p} k\right)=\hat{\mu}_{n-1}\left(\ell^{p} k\right),
\end{aligned}
$$

for $0 \leqslant n \leqslant m$. Therefore, from (11), it follows that

$$
m(-1)^{m-1} \hat{\mu}_{0}\left(\ell^{p} k\right)-\hat{\mu}_{m}\left(\ell^{p+1} k\right)=(-1)^{m+1} \hat{\mu}_{0}\left(\ell^{p+1} k\right) .
$$

Hence,

$$
m \leqslant \frac{\left|\hat{\mu}_{m}\left(\ell^{p+1} k\right)\right|+\left|\hat{\mu}_{0}\left(\ell^{p+1} k\right)\right|}{\left|\hat{\mu}_{0}\left(\ell^{p} k\right)\right|}
$$

and by (10) we obtain an upper bound for $m$ :

$$
m \leqslant \frac{\|\tau\|_{L}+4 k \ell^{p+1}\left|\hat{\tau}\left(\ell^{p+1} k\right)\right|}{4 k \ell^{p+1}\left|\hat{\tau}\left(\ell^{p} k\right)\right|} .
$$

Below we record the explicit bound obtained in the previous proof.

Corollary 5.7. Let $\tau: \mathbb{R} / \mathbb{Z} \rightarrow \mathbb{R}$ be a Lipschitz function such that:

$$
\begin{aligned}
& \hat{\tau}\left(\ell^{j} k\right)=0 \quad \text { for } 0 \leqslant j<p, \\
& \hat{\tau}\left(\ell^{p} k\right) \neq 0
\end{aligned}
$$

for some integers $k \in \mathbb{Z}$ and $p \geqslant 0$ with $\ell \nmid k$. If there exists $m \geqslant 0$ and a continuous function $\mu: \mathbb{R} / \mathbb{Z} \rightarrow \mathbb{R}$ such that

$$
\mathcal{L}_{1}^{m} \mu=\tau .
$$

Then

$$
m \leqslant \frac{\|\tau\|_{L}+4 k \ell^{p+1}\left|\hat{\tau}\left(\ell^{p+1} k\right)\right|}{4 k \ell^{p+1}\left|\hat{\tau}\left(\ell^{p} k\right)\right|} .
$$

Main Lemma. Let $\tau: \mathbb{R} / \mathbb{Z} \rightarrow \mathbb{R}$ be a non-constant Lipschitz function. Then the set $\mathcal{J}_{\tau}^{\prime}$ is finite, counting multiplicities.

Moreover, consider the finite collection $0<\lambda_{1}, \ldots, \lambda_{m} \leqslant 1$ consisting of elements of $\mathcal{J}_{\tau}^{\prime}$ where the number of repetitions of each element of $\mathcal{J}_{\tau}^{\prime}$ coincides with its multiplicity. Then there exists a Lipschitz function $\mu: \mathbb{R} / \mathbb{Z} \rightarrow \mathbb{R}$ such that

$$
\tau=\mathcal{L}_{\lambda_{1}} \circ \cdots \circ \mathcal{L}_{\lambda_{m}} \mu
$$

and such that $\mu \neq \mathcal{L}_{\lambda} \rho$ for all $\lambda \in(0,1]$ and all Lipschitz functions $\rho: \mathbb{R} / \mathbb{Z} \rightarrow \mathbb{R}$. Furthermore, the function $\mu$ is uniquely determined by this property, up to an additive constant. If $\int \tau=0$, then $\mu$ may be chosen so that $\int \mu=0$. 
Proof. By Lemma 5.2 and Remark 5.3 it is sufficient to show that $\mathcal{J}_{\tau}^{\prime}$ is finite, counting multiplicities. If $\operatorname{mult}_{\tau}(1)>0$, then there exists a Lipschitz function $\mu: \mathbb{R} / \mathbb{Z} \rightarrow \mathbb{R}$ such that $\mathcal{L}_{1}^{\text {mult }_{\tau}(1)} \mu=\tau$ (Lemma 5.6). By Lemma 5.2 it follows that $\operatorname{mult}_{\mu}(1)=0$ and that for every $\lambda \in(0,1)$ we have mult ${ }_{\mu}(\lambda)=$ mult $_{\tau}(\lambda)$. So, replacing $\tau$ by $\mu$ if necessary, we reduce to the case when $\operatorname{mult}_{\tau}(1)=0$. Then Lemma 5.5 implies that there exists $\lambda_{0} \in(0,1)$ such that $\mathcal{J}_{\tau}^{\prime}$ is contained in $\left(0, \lambda_{0}\right]$.

We proceed by contradiction and suppose $\mathcal{J}_{\tau}^{\prime}$ is infinite or that it contains an element with infinite multiplicity. In both cases, for all $n \geqslant 1$ there exist $\lambda_{n} \in\left(0, \lambda_{0}\right]$ and a Lipschitz function $\mu_{n}: \mathbb{R} / \mathbb{Z} \rightarrow \mathbb{R}$ such that

$$
\mathcal{L}_{\lambda_{1}} \circ \cdots \circ \mathcal{L}_{\lambda_{n}} \mu_{n}=\tau \text {. }
$$

Lemma 5.4 implies that for every $n \geqslant 1$ we have,

$$
\|\tau\|_{L} \geqslant\left(\ell-\lambda_{1}\right) \cdots\left(\ell-\lambda_{n}\right)\left\|\mu_{n}\right\|_{L},
$$

so that $\left\|\mu_{n}\right\|_{L} \leqslant\|\tau\|_{L}$. Therefore, for all $n \geqslant 1$ and $k \in \mathbb{Z}$ different from 0 we have,

$$
\left|\hat{\mu}_{n}(k)\right| \leqslant\|\tau\|_{L},
$$

where $\hat{\mu}_{n}(k)$ denotes the $k$-th Fourier coefficient of $\mu_{n}$.

Setting $\mu_{0}=\tau$, Lemma 5.2(3) implies that for all $n \geqslant 1$ we have,

$$
\mathcal{L}_{\lambda_{n}} \mu_{n}=\mu_{n-1} .
$$

Hence, $\mu_{n}(\ell \theta)-\lambda_{n} \mu_{n}(\theta)=\mu_{n-1}(\theta)$ and for all $k \in \mathbb{Z}$ such that $\ell \nmid k$ we have,

$$
\begin{aligned}
& \hat{\mu}_{n}\left(\ell^{j-1} k\right)-\lambda_{n} \hat{\mu}_{n}\left(\ell^{j} k\right)=\hat{\mu}_{n-1}\left(\ell^{j} k\right) \quad \text { for } j \geqslant 1, \\
& -\lambda_{n} \hat{\mu}_{n}(k)=\hat{\mu}_{n-1}(k) .
\end{aligned}
$$

Fix $k \neq 0$ such that $\ell$ does not divide $k$. We show by induction on $j \geqslant 0$ that $\hat{\mu}_{n}\left(\ell^{j} k\right)=0$ for all $n \geqslant 0$.

For $j=0$, by Eq. (16), $\hat{\mu}_{0}(k)=(-1)^{n} \lambda_{1} \cdots \lambda_{n} \hat{\mu}_{n}(k)$. Since the $\hat{\mu}_{n}(k)$ are uniformly bounded, it follows that $\hat{\mu}_{0}(k)=0$ and therefore $\hat{\mu}_{n}(k)=0$ for all $n$.

Let $j \geqslant 1$ and suppose that $\hat{\mu}_{n}\left(\ell^{j-1} k\right)=0$ for all $n \geqslant 0$. It follows from Eq. (15) that $-\lambda_{n} \hat{\mu}_{n}\left(\ell^{j} k\right)=\hat{\mu}_{n-1}\left(\ell^{j} k\right)$. Hence, $\hat{\mu}_{0}\left(\ell^{j} k\right)=(-1)^{n} \lambda_{1} \cdots \lambda_{n} \hat{\mu}_{n}\left(\ell^{j} k\right)$. Again using that $\hat{\mu}_{n}\left(\ell^{j} k\right)$ are uniformly bounded, it follows that $\hat{\mu}_{n}\left(\ell^{j} k\right)=0$ for all $n$.

We conclude that $\tau=\mu_{0}$ is constant which is a contradiction.

\subsection{Theorems 1 and 3}

We now state and prove Theorem 3 which is a stronger version of Theorem 1 . Recall that $\mathcal{J}_{\tau}$ denotes the set of $\lambda \in(0,1)$ such that $A_{\lambda, \tau}$ is a Jordan curve.

Theorem 3. Suppose that $\tau: \mathbb{R} / \mathbb{Z} \rightarrow \mathbb{R}$ is a non-constant Lipschitz. Then there exists a Lipschitz map $\mu: \mathbb{R} / \mathbb{Z} \rightarrow \mathbb{R}$ satisfying the following properties:

(1) $\mathcal{J}_{\mu}=\emptyset$ and for all $\lambda \in(0,1) \backslash \mathcal{J}_{\tau}$ the maps $A_{\lambda, \tau}$ and $A_{\lambda, \mu}$ are topologically conjugate.

(2) $\int \mu=0$ and $\mu$ is not cohomologous to 0 .

Lemma 5.8. Let $\tau: \mathbb{R} / \mathbb{Z} \rightarrow \mathbb{R}$ be a map of class $\mathcal{C}$ where $\mathcal{C}$ is the class of Lipschitz or $C^{r}$ maps for some $r \in$ $(0, \infty] \cup\{\omega\}$. Then:

(1) For all $c \in \mathbb{R}$ and $0<\lambda<1$, the maps $A_{\lambda, \tau}$ and $A_{\lambda, \tau+c}$ are conjugate via an affine map.

(2) If $\mu \circ \mathbf{m}_{\ell}-\lambda_{0} \mu=\tau$ for some continuous map $\mu: \mathbb{R} / \mathbb{Z} \rightarrow \mathbb{R}$ and $\lambda_{0} \in(0,1]$, then $A_{\lambda, \tau}$ and $A_{\lambda, \mu}$ are $\mathcal{C}$-conjugate for all $\lambda \neq \lambda_{0}$. 
Proof. For (1) note that $A_{\lambda, \tau} \circ h=h \circ A_{\lambda, \tau+c}$ where $h(\theta, t)=(\theta, t-c /(1-\lambda))$.

For (2), if $\mu \circ \mathbf{m}_{\ell}-\lambda_{0} \mu=\tau$ for some continuous map $\mu$, then $\mu$ is automatically of class $\mathcal{C}$ and $h(\theta, t)=$ $\left(\theta,\left(\lambda-\lambda_{0}\right) t+\mu(\theta)\right)$ is such that $h \circ A_{\lambda, \mu}=A_{\lambda, \tau} \circ h$ for all $\lambda \neq \lambda_{0}$.

Proof of Theorem 3. By Lemma 5.8(1), after replacing $\tau$ by $\tau-\int \tau$, we may assume that $\int \tau=0$. In view of Proposition 3.1 and Lemma 5.8(2), statements (1) and (2) hold for the function $\mu: \mathbb{R} / \mathbb{Z} \rightarrow \mathbb{R}$ given by the Main Lemma.

Proof of Theorem 1. For (1) just note that the Main Lemma implies that $\mathcal{J}_{\tau}$ is finite. For (2) let $\mu: \mathbb{R} / \mathbb{Z} \rightarrow \mathbb{R}$ be as in Theorem 3 and apply Proposition 4.6.

\section{Continuity of the upper and lower boundaries}

In this section we show that for every map that is sufficiently (Lipschitz) close to $A_{\lambda, \tau}$ has an attractor with well defined upper and lower boundaries. Here $A$ need not be a skew product map.

The aim of this section is to show that the upper and lower boundaries of $\Omega_{\lambda, \tau}$ vary continuously under Lipschitz perturbations of $A_{\lambda, \tau}$.

Given an open set $U \subset \mathbb{R} / \mathbb{Z} \times \mathbb{R}$ with compact closure we denote by $\operatorname{Lip}(\bar{U}, \mathbb{R} / \mathbb{Z} \times \mathbb{R})$ the set formed by all the Lipschitz maps $F: \bar{U} \rightarrow \mathbb{R} / \mathbb{Z} \times \mathbb{R}$ endowed with the Lipschitz metric dist ${ }_{L}$. More precisely,

$$
\operatorname{dist}_{L}\left(F_{0}, F_{1}\right)=\left\|F_{0}-F_{1}\right\|_{\infty}+\left\|f_{0}-f_{1}\right\|_{L}+\left\|g_{0}-g_{1}\right\|_{L}
$$

where $F_{i}(\theta, t)=\left(f_{i}(\theta, t), g_{i}(\theta, t)\right)$ for $i=0,1$.

Note that the $\operatorname{set} \operatorname{Lip}(\bar{U}, U)$ of all $F \in \operatorname{Lip}(\bar{U}, \mathbb{R} / \mathbb{Z} \times \mathbb{R})$ such that $F(\bar{U}) \subset U$ is open in $\operatorname{Lip}(\bar{U}, \mathbb{R} / \mathbb{Z} \times \mathbb{R})$.

Definition 6.1. Suppose that $U \subset \mathbb{R} / \mathbb{Z} \times \mathbb{R}$ is an open set with compact closure. For any $F \in \operatorname{Lip}(\bar{U}, U)$ we let $\Omega_{F}=\bigcap_{n \geqslant 0} F^{n}(\bar{U})$ be the attractor of $F$. The upper and lower boundaries of $\Omega_{F}$ are the graphs of

$$
\rho_{F}^{ \pm}: \mathbb{R} / \mathbb{Z} \rightarrow \mathbb{R} \cup\{ \pm \infty\}
$$

where

$$
\begin{aligned}
& \rho_{F}^{+}(\theta)=\sup \left\{t \in \mathbb{R} \mid(\theta, t) \in \Omega_{F}\right\}, \\
& \rho_{F}^{-}(\theta)=\inf \left\{t \in \mathbb{R} \mid(\theta, t) \in \Omega_{F}\right\}
\end{aligned}
$$

if $\Omega_{F} \cap(\{\theta\} \times \mathbb{R}) \neq \emptyset$ and $\rho^{ \pm}(\theta)=\mp \infty$ otherwise.

We may now state the main result of this section.

Proposition 6.2. Consider $\lambda \in(0,1)$ and a Lipschitz function $\tau: \mathbb{R} / \mathbb{Z} \rightarrow \mathbb{R}$. Let $T_{0} \in \mathbb{R}$ be such that $A_{\lambda, \tau}\left(\overline{U_{0}}\right) \subset U_{0}$ where $U_{0}=\mathbb{R} / \mathbb{Z} \times\left(-T_{0}, T_{0}\right)$. Given $\epsilon>0$ there exists a neighborhood $\mathcal{U} \subset \operatorname{Lip}\left(\overline{U_{0}}, U_{0}\right)$ of $A_{\lambda, \tau}$ such that for all $F \in \mathcal{U}$ the following hold:

(1) $\rho_{F}^{ \pm}: \mathbb{R} / \mathbb{Z} \rightarrow \mathbb{R}$ are well defined Lipschitz functions.

(2) $\left\|\rho_{F}^{ \pm}-\rho_{A_{\lambda, \tau}}^{ \pm}\right\|_{\infty}<\epsilon$. 


\subsection{Lipschitz maps in $\mathbb{R}^{2}$}

In this subsection we consider $\bar{V}=\mathbb{R} \times\left[-T_{0}, T_{0}\right] \subset \mathbb{R}^{2}$ and study the action of Lipschitz maps $\widetilde{F}: \bar{V} \rightarrow \mathbb{R}^{2}$ on the graphs of Lipschitz functions $\tilde{\rho}: \mathbb{R} \rightarrow\left[-T_{0}, T_{0}\right]$. The results obtained here will be applied in the proof of Proposition 6.2 to the lift of maps $F$ which are Lipschitz close to $A_{\lambda, \tau}$.

Throughout this subsection we consider a map:

$$
\begin{aligned}
\widetilde{F}: \bar{V} & \rightarrow \mathbb{R}^{2}, \\
(s, t) & \mapsto(\tilde{f}(s, t), \tilde{g}(s, t))
\end{aligned}
$$

and suppose that there exist positive constants $\ell_{0}, \lambda_{0}, C_{1,2}$ and $C_{2,1}$ such that:

$$
\begin{aligned}
& \tilde{f}\left(s_{0}, t\right)-\tilde{f}\left(s_{1}, t\right) \geqslant \ell_{0}\left(s_{0}-s_{1}\right), \\
& \left|\tilde{g}\left(s_{0}, t\right)-\tilde{g}\left(s_{1}, t\right)\right| \leqslant C_{2,1}\left|s_{0}-s_{1}\right|, \\
& \left|\tilde{f}\left(s, t_{0}\right)-\tilde{f}\left(s, t_{1}\right)\right| \leqslant C_{1,2}\left|t_{0}-t_{1}\right|, \\
& \left|\tilde{g}\left(s, t_{0}\right)-\tilde{g}\left(s, t_{1}\right)\right| \leqslant \lambda_{0}\left|t_{0}-t_{1}\right|,
\end{aligned}
$$

for all $s_{0}>s_{1}$ and $t, t_{0}, t_{1} \in\left[-T_{0}, T_{0}\right]$.

The above conditions are satisfied by the lift $\tilde{A}_{\lambda, \tau}(s, t)=(\ell s, \lambda t+\tau \circ \pi(s))$ of $A_{\lambda, \tau}$ where $\tau: \mathbb{R} / \mathbb{Z} \rightarrow \mathbb{R}$ is Lipschitz and $\lambda \in(0,1)$. Observe that in this case we may choose $\ell_{0}=\ell, C_{2,1}=\|\tau\|_{L}, C_{1,2}=0$, and $\lambda_{0}=\lambda$.

Lemma 6.3. If $\tilde{\gamma} \subset \bar{V}$ is the graph of a Lipschitz function $\tilde{\rho}: \mathbb{R} \rightarrow\left[-T_{0}, T_{0}\right]$ such that $\|\tilde{\rho}\|_{L}<\ell_{0} C_{1,2}^{-1}$, then $\widetilde{F}(\tilde{\gamma})$ is the graph of a $C$-Lipschitz function $\mathcal{T}_{\widetilde{F}}(\tilde{\rho}): \mathbb{R} \rightarrow \mathbb{R}$, where

$$
C=\frac{C_{2,1}+\lambda_{0}\|\tilde{\rho}\|_{L}}{\ell_{0}-C_{1,2}\|\tilde{\rho}\|_{L}} .
$$

Proof. Suppose that $s_{0}>s_{1}$. For $i=0,1$, let $t_{i}=\tilde{\rho}\left(s_{i}\right)$ and $\left(s_{i}^{\prime}, t_{i}^{\prime}\right)=\widetilde{F}\left(s_{i}, t_{i}\right)$. Then we have that:

$$
\begin{aligned}
s_{0}^{\prime}-s_{1}^{\prime} & =\tilde{f}\left(s_{0}, t_{0}\right)-\tilde{f}\left(s_{1}, t_{1}\right)=\left(\tilde{f}\left(s_{0}, t_{0}\right)-\tilde{f}\left(s_{0}, t_{1}\right)\right)+\left(\tilde{f}\left(s_{0}, t_{1}\right)-\tilde{f}\left(s_{1}, t_{1}\right)\right) \\
& \geqslant-C_{1,2}\left|t_{0}-t_{1}\right|+\ell_{0}\left(s_{0}-s_{1}\right)>-\ell_{0}\left|s_{0}-s_{1}\right|+\ell_{0}\left(s_{0}-s_{1}\right)=0 .
\end{aligned}
$$

In particular, $\widetilde{F}(\tilde{\gamma})$ is the graph of some function. Also,

$$
\left|t_{0}^{\prime}-t_{1}^{\prime}\right|=\left|\tilde{g}\left(s_{0}, t_{0}\right)-\tilde{g}\left(s_{1}, t_{1}\right)\right|=\left|\tilde{g}\left(s_{0}, t_{0}\right)-\tilde{g}\left(s_{0}, t_{1}\right)+\tilde{g}\left(s_{0}, t_{1}\right)-\tilde{g}\left(s_{1}, t_{1}\right)\right| \leqslant \lambda_{0}\left|t_{0}-t_{1}\right|+C_{2,1}\left|s_{0}-s_{1}\right| .
$$

Hence,

$$
\frac{\left|t_{0}^{\prime}-t_{1}^{\prime}\right|}{\left|s_{0}^{\prime}-s_{1}^{\prime}\right|} \leqslant \frac{\lambda_{0}\left|t_{0}-t_{1}\right|+C_{2,1}\left|s_{0}-s_{1}\right|}{-C_{1,2}\left|t_{0}-t_{1}\right|+\ell_{0}\left|s_{0}-s_{1}\right|} \leqslant \frac{C_{2,1}+\lambda_{0}\|\tilde{\rho}\|_{L}}{\ell_{0}-C_{1,2}\|\tilde{\rho}\|_{L}}
$$

and the lemma follows.

Definition 6.4. We say that a Lipschitz map $\widetilde{F}: \bar{V} \rightarrow \mathbb{R}^{2}$ preserves $C$-Lipschitz graphs with constants $\ell_{0}, \lambda_{0}, C_{1,2}$ and $C_{2,1}$ if (17)-(20) hold and

$$
\frac{C_{2,1}+\lambda_{0} C}{\ell_{0}-C_{1,2} C} \leqslant C<\frac{\ell_{0}}{C_{1,2}} \text {. }
$$

In particular, if $\widetilde{F}: \bar{V} \rightarrow \bar{V} \subset \mathbb{R}^{2}$ preserves $C$-Lipschitz graphs, then $\mathcal{T}_{\widetilde{F}}$ acts on the set of $C$-Lipschitz functions $\rho: \mathbb{R} \rightarrow\left[-T_{0}, T_{0}\right]$ (see Lemma 6.3).

Now we compute a Lipschitz constant for $\mathcal{T}_{\widetilde{F}}$ with respect to the $C^{0}$-norm. 
Lemma 6.5. Let $C>0$ be such that $C<\ell_{0} C_{1,2}^{-1}$. Suppose that $\tilde{\rho}_{i}: \mathbb{R} \rightarrow\left[-T_{0}, T_{0}\right]$ are $C$-Lipschitz functions where $i=0,1$. Then

$$
\left\|\mathcal{T}_{\widetilde{F}}\left(\tilde{\rho}_{0}\right)-\mathcal{T}_{\widetilde{F}}\left(\tilde{\rho}_{1}\right)\right\|_{\infty} \leqslant \frac{\lambda_{0} \ell_{0}+C_{2,1} C_{1,2}}{\ell_{0}-C_{1,2} C}\left\|\tilde{\rho}_{0}-\tilde{\rho}_{1}\right\|_{\infty}
$$

Proof. Consider $s^{\prime} \in \mathbb{R}$. For $i=0,1$, let $\tilde{\rho}_{i}^{\prime}=\mathcal{T}_{\widetilde{F}}\left(\tilde{\rho}_{i}\right), t_{i}^{\prime}=\tilde{\rho}_{i}^{\prime}\left(s^{\prime}\right)$. Also we let $\left(s_{i}, t_{i}\right)$ be the points in the graph of $\tilde{\rho}_{i}$ such that $\widetilde{F}\left(s_{i}, t_{i}\right)=\left(s^{\prime}, t_{i}^{\prime}\right)$. We may assume that $s_{1}>s_{0}$.

We must find an upper bound for $\left|t_{1}^{\prime}-t_{0}^{\prime}\right|$ in terms of $\left|\hat{t}_{1}-t_{0}\right|$ where $\hat{t}_{1}=\tilde{\rho}_{1}\left(s_{0}\right)$.

Since

$$
0=\tilde{f}\left(s_{0}, t_{0}\right)-\tilde{f}\left(s_{1}, t_{1}\right)=\tilde{f}\left(s_{0}, t_{0}\right)-\tilde{f}\left(s_{1}, t_{0}\right)+\tilde{f}\left(s_{1}, t_{0}\right)-\tilde{f}\left(s_{1}, t_{1}\right),
$$

we have that

$$
\tilde{f}\left(s_{1}, t_{0}\right)-\tilde{f}\left(s_{0}, t_{0}\right)=\left|\tilde{f}\left(s_{1}, t_{1}\right)-\tilde{f}\left(s_{1}, t_{0}\right)\right| .
$$

Therefore,

$$
\ell_{0}\left(s_{1}-s_{0}\right) \leqslant C_{1,2}\left|t_{1}-t_{0}\right| .
$$

Also,

$$
\begin{aligned}
\left|t_{1}^{\prime}-t_{0}^{\prime}\right| & =\left|\tilde{g}\left(s_{0}, t_{0}\right)-\tilde{g}\left(s_{1}, t_{1}\right)\right| \leqslant\left|\tilde{g}\left(s_{0}, t_{0}\right)-\tilde{g}\left(s_{0}, t_{1}\right)\right|+\left|\tilde{g}\left(s_{0}, t_{1}\right)-\tilde{g}\left(s_{1}, t_{1}\right)\right| \\
& \leqslant \lambda_{0}\left|t_{0}-t_{1}\right|+C_{2,1}\left|s_{0}-s_{1}\right| .
\end{aligned}
$$

Since

$$
\left|t_{1}-t_{0}\right| \leqslant\left|\hat{t}_{1}-t_{0}\right|+\left|\hat{t}_{1}-t_{1}\right| \leqslant\left|\hat{t}_{1}-t_{0}\right|+C\left|s_{0}-s_{1}\right|,
$$

it follows from (21) that

$$
\left|t_{1}-t_{0}\right| \leqslant\left|\hat{t}_{1}-t_{0}\right|+C C_{1,2} \ell_{0}^{-1}\left|t_{1}-t_{0}\right| .
$$

Hence,

$$
\left|t_{1}-t_{0}\right| \leqslant\left(1-C C_{1,2} \ell_{0}^{-1}\right)^{-1}\left|\hat{t}_{1}-t_{0}\right| .
$$

Combining (21) and (22) we obtain

$$
\left|t_{1}^{\prime}-t_{0}^{\prime}\right| \leqslant\left(\lambda_{0}+C_{2,1} C_{1,2} \ell_{0}^{-1}\right)\left|t_{1}-t_{0}\right| .
$$

The lemma now follows directly from (23) and (24).

Lemma 6.6. For $i=0$, 1 , let $\widetilde{F}_{i}(s, t)=\left(\tilde{f}_{i}(s, t), \tilde{g}_{i}(s, t)\right)$ be maps in $\operatorname{Lip}\left(\bar{V}, \mathbb{R}^{2}\right)$ such that $\widetilde{F}_{i}$ preserve C-Lipschitz graphs with constants $\ell_{0}, \lambda_{0}, C_{1,2}$ and $C_{2,1}$. If $\left\|\widetilde{F}_{0}-\widetilde{F}_{1}\right\|_{\infty}<\epsilon$, then

$$
\left\|\mathcal{T}_{\widetilde{F}_{0}}(\tilde{\rho})-\mathcal{T}_{\widetilde{F}_{1}}(\tilde{\rho})\right\|_{\infty} \leqslant(1+C) \epsilon
$$

for all C-Lipschitz functions $\tilde{\rho}: \mathbb{R} \rightarrow\left[-T_{0}, T_{0}\right]$.

Proof. Consider $s_{0} \in \mathbb{R}$ and let $t_{0}=\tilde{\rho}\left(s_{0}\right)$. Also let $\left(s_{i}^{\prime}, t_{i}^{\prime}\right)=F_{i}\left(s_{0}, t_{0}\right)$ where $i=0,1$. It follows that

$$
\left|\tilde{\rho}_{1}^{\prime}\left(s_{1}^{\prime}\right)-\tilde{\rho}_{0}^{\prime}\left(s_{1}^{\prime}\right)\right| \leqslant\left|\tilde{\rho}_{1}^{\prime}\left(s_{1}^{\prime}\right)-\tilde{\rho}_{0}^{\prime}\left(s_{0}^{\prime}\right)\right|+\left|\tilde{\rho}_{0}^{\prime}\left(s_{0}^{\prime}\right)-\tilde{\rho}_{0}^{\prime}\left(s_{1}^{\prime}\right)\right| \leqslant \epsilon+C\left|s_{0}^{\prime}-s_{1}^{\prime}\right| \leqslant \epsilon+C \epsilon
$$

where $\mathcal{T}_{F_{i}}(\tilde{\rho})=\tilde{\rho}_{i}^{\prime}$. 


\subsection{The upper and lower boundaries as fixed points}

Throughout this subsection we let $T_{0}>0$ and $U_{0}=\mathbb{R} / \mathbb{Z} \times\left(-T_{0}, T_{0}\right)$. Here we consider a map $F \in \operatorname{Lip}\left(\overline{U_{0}}, U_{0}\right)$ and show that under certain conditions the upper and lower boundaries of the attractor $\Omega_{F}$ are Lipschitz graphs.

Lemma 6.7. Let $F \in \operatorname{Lip}\left(\overline{U_{0}}, U_{0}\right)$ be locally an orientation preserving homeomorphism which acts as multiplication by $\ell \geqslant 1$ on the first homology of $\mathbb{R} / \mathbb{Z} \times\left(-T_{0}, T_{0}\right)$. Denote by $\widetilde{F}: \mathbb{R} \times\left[-T_{0}, T_{0}\right] \rightarrow \mathbb{R} / \mathbb{Z} \times\left(-T_{0}, T_{0}\right)$ a lift of $F$ to the universal cover and suppose that $\widetilde{F}$ preserves $C$-Lipschitz maps with constants $\ell_{0}, \lambda_{0}, C_{1,2}, C_{2,1}$. Assume that:

$$
\frac{\lambda_{0} \ell_{0}+C_{2,1} C_{1,2}}{\ell_{0}-C_{1,2} C}<1
$$

Let

$$
\mathcal{T}_{F}^{+}(\tilde{\rho})(s)=\max \left\{\mathcal{T}_{\widetilde{F}}(\tilde{\rho})(s), \mathcal{T}_{\widetilde{F}}(\tilde{\rho})(s+1), \ldots, \mathcal{T}_{\widetilde{F}}(\tilde{\rho})(s+\ell-1)\right\}
$$

and

$$
\mathcal{T}_{F}^{-}(\tilde{\rho})(s)=\min \left\{\mathcal{T}_{\widetilde{F}}(\tilde{\rho})(s), \mathcal{T}_{\widetilde{F}}(\tilde{\rho})(s+1), \ldots, \mathcal{T}_{\widetilde{F}}(\tilde{\rho})(s+\ell-1)\right\} .
$$

Then $\mathcal{T}_{F}^{ \pm}$are contractions in the space of $C$-Lipschitz functions $\tilde{\rho}: \mathbb{R} \rightarrow\left[-T_{0}, T_{0}\right]$ endowed with $\|\cdot\|_{\infty}$. The fixed points $\tilde{\rho}_{F}^{ \pm}$of $\mathcal{T}_{F}^{ \pm}$are 1-periodic and the graphs of

$$
\begin{aligned}
\rho_{F}^{ \pm}: \mathbb{R} / \mathbb{Z} & \rightarrow \mathbb{R}, \\
\theta=\pi(s) & \mapsto \tilde{\rho}_{F}^{ \pm}(s)
\end{aligned}
$$

are the upper and lower boundaries of the attractor $\Omega_{F}$.

Proof. Since the maximum and minimum of $C$-Lipschitz functions are also $C$-Lipschitz, from Lemma 6.3 it follows that $\mathcal{T}_{F}^{ \pm}(\tilde{\rho})$ are $C$-Lipschitz whenever $\tilde{\rho}$ is $C$-Lipschitz. By Lemma 6.5, the operators $\mathcal{T}_{F}^{ \pm}$act as contraction maps. Our hypothesis that $F$ acts as multiplication by $\ell$ on the first homology group translates to the universal cover as $\widetilde{F}(s+1, t)=\widetilde{F}(s, t)+(\ell, 0)$. It follows that $\mathcal{T}_{F}^{ \pm}$preserve the closed subset of 1-periodic $C$-Lipschitz function. Therefore, the fixed points $\tilde{\rho}_{F}^{ \pm}$are 1-periodic and we let $\rho_{F}^{ \pm}(\pi(s))=\tilde{\rho}_{F}^{ \pm}(s)$.

We now show that $\rho_{F}^{+}$is the upper boundary of $\Omega_{F}$. Note that the graph $\gamma_{F}^{+}$of $\rho_{F}^{+}$is invariant under $F$. That is $F\left(\gamma_{F}^{+}\right) \supset \gamma_{F}^{+}$. Therefore $\gamma_{F}^{+} \subset \Omega_{F}$. It is sufficient to show that $\Omega_{F}$ is below $\gamma_{F}^{F}$. For this let $\tilde{\rho}_{0}$ be the constant function $T_{0}$ on $\mathbb{R}$ and let $\tilde{\rho}_{n}=\mathcal{T}_{F}^{+}\left(\tilde{\rho}_{0}\right)$. From our previous discussion and the fact that $\tilde{\rho}_{0}$ is 1-periodic, we conclude that $\tilde{\rho}_{n}$ projects to a function $\rho_{n}: \mathbb{R} / \mathbb{Z} \rightarrow \mathbb{R}$ with graph $\gamma_{n}$. Since $F$ is locally an orientation preserving homeomorphism, $F^{n}\left(\overline{U_{0}}\right)$ has as upper boundary the curve $\gamma_{n}$. Taking into consideration that $\rho_{n}$ converges to $\rho$, it follows that $\cap F^{n}\left(\overline{U_{0}}\right)$ is below $\gamma_{F}^{+}$. A similar argument shows that $\rho_{F}^{-}$is the lower boundary of $\Omega_{F}$.

The analogue of Lemma 4.1 also holds in this context. More precisely:

Lemma 6.8. Assume that $F \in \operatorname{Lip}\left(\overline{U_{0}}, U_{0}\right)$ is locally an orientation preserving homeomorphism such that the upper and lower boundaries of $\Omega_{F}$ are the graphs $\gamma_{F}^{ \pm}$of functions $\rho_{F}^{ \pm}: \mathbb{R} / \mathbb{Z} \rightarrow \mathbb{R}$. If for all $\theta \in \mathbb{R} / \mathbb{Z}$

$$
\min \left\{t \mid(\theta, t) \in F\left(\gamma_{F}^{+}\right)\right\}>\max \left\{t \mid(\theta, t) \in F\left(\gamma_{F}^{-}\right)\right\},
$$

then $\Omega_{F}$ is a closed topological annulus. Moreover, if $\ell=2$ and for some $\theta \in \mathbb{R} / \mathbb{Z}$

$$
\min \left\{t \mid(\theta, t) \in F\left(\gamma_{F}^{+}\right)\right\}<\max \left\{t \mid(\theta, t) \in F\left(\gamma_{F}^{-}\right)\right\},
$$

then $\Omega_{F}$ is not a closed topological annulus.

We omit the proof of this lemma since it is very similar to that of Lemma 4.1. 


\subsection{Proof of Proposition 6.2}

Since Lipschitz perturbations of bilipschitz maps are bilipschitz, all $F$ in a sufficiently small neighborhood $\mathcal{U}$ of $A_{\lambda, \tau}$ in $\operatorname{Lip}\left(\overline{U_{0}}, U_{0}\right)$ are locally orientation preserving homeomorphisms. Consider a small real number $\delta>0$ and shrink $\mathcal{U}$, if necessary, so that for all $F \in \mathcal{U}$ there exists a unique lift $\widetilde{F}: \mathbb{R} \times\left[-T_{0}, T_{0}\right] \rightarrow \mathbb{R}^{2}$ of $F$ such that $\left\|\widetilde{F}-\tilde{A}_{\lambda, \tau}\right\|_{\infty}<\delta$, where $\tilde{A}_{\lambda, \tau}(s, t)=(\ell s, \lambda t+\tau \circ \pi(s))$. After further shrinking of $\mathcal{U}$, if necessary, we may assume that there exist positive constants: $\ell_{0}$ slightly smaller than $\ell, \lambda_{0}$ close to $\lambda, C_{2,1}$ close to $\|\tau\|_{L}$, and $C_{1,2}$ sufficiently small such that for all $F \in \mathcal{U}$ the corresponding lift $\widetilde{F}$ preserves $C$-Lipschitz graphs with constants $\ell_{0}, \lambda_{0}, C_{2,1}, C_{1,2}$ and the following inequalities also hold:

$$
\begin{aligned}
& C>(\ell-\lambda)^{-1}\|\tau\|_{L} \geqslant\left\|\rho_{A_{\lambda, \tau}}^{ \pm}\right\|_{L}, \\
& \hat{\lambda}=\frac{\lambda_{0} \ell_{0}+C_{2,1} C_{1,2}}{\ell_{0}-C_{1,2} C}<1 .
\end{aligned}
$$

Let $\tilde{\rho}^{+}$be the lift of $\rho_{A_{\lambda, \tau}}^{+}$. By Lemma 6.6,

$$
\left\|\left(\mathcal{T}_{F}^{+}\right)^{n}\left(\tilde{\rho}^{+}\right)-\tilde{\rho}^{+}\right\|_{\infty} \leqslant \sum_{k=0}^{n-1}\left\|\left(\mathcal{T}_{F}^{+}\right)^{k+1}\left(\tilde{\rho}^{+}\right)-\left(\mathcal{T}_{F}^{+}\right)^{k}\left(\tilde{\rho}^{+}\right)\right\|_{\infty} \leqslant\left(\sum_{k=0}^{n-1} \hat{\lambda}^{k}\right)(1+C) \delta \leqslant \frac{1+C}{1-\hat{\lambda}} \delta .
$$

Choosing $\delta>0$ so that $(1+C) /(1-\hat{\lambda}) \delta=\epsilon$ it follows that $\tilde{\rho}_{F}^{+}=\lim \left(\mathcal{T}_{F}^{+}\right)^{n}\left(\tilde{\rho}^{+}\right)$is $\epsilon$-close to $\tilde{\rho}^{+}$. Similarly, we obtain that $\tilde{\rho}_{F}^{-}$is $\epsilon$-close to $\tilde{\rho}^{-}$.

\section{Examples}

\subsection{Perturbation of affine maps with annular attractors}

In the previous section we showed that the upper and lower boundaries of the attractor $\Omega_{\lambda, \tau}$ of an affine map $A_{\lambda, \tau}$ move continuously under Lipschitz perturbations. In view of Lemma 4.1, Remark 4.8 and Theorem 3 we obtain the following result.

Proposition 7.1. Let $\tau: \mathbb{R} / \mathbb{Z} \rightarrow \mathbb{R}$ be a Lipschitz function. Then there exists $\lambda_{0} \in(1 / \ell, 1)$ such that for any $\lambda \in$ $\left[\lambda_{0}, 1\right)$ and for all $F$ in an appropriate neighborhood $\mathcal{U}$ of $A_{\lambda, \tau}$ in $\operatorname{Lip}(\bar{U}, U)$ we have that $\Omega_{F}$ is an annulus where $U=\mathbb{R} / \mathbb{Z} \times\left(-T_{0}, T_{0}\right)$ is such that $A_{\lambda, \tau} \in \operatorname{Lip}(\bar{U}, U)$.

We will apply the above proposition to exhibit annular attractors in an explicit family of endomorphisms of $\mathbb{C}^{*}=\mathbb{C} \backslash\{0\}$. More precisely, we consider the family

$$
\begin{aligned}
f_{\lambda, c}: \mathbb{C}^{*} & \rightarrow \mathbb{C}, \\
z & \mapsto(\lambda|z|+1-\lambda) \frac{z^{2}}{|z|^{2}}+c,
\end{aligned}
$$

where $c \in \mathbb{C}$ and $\lambda \in(0,1)$. Observe that $f_{\lambda, 0}\left(\mathbb{C}^{*}\right)=\{z \in \mathbb{C}|| z \mid>1-\lambda\} \subset \mathbb{C}^{*}$ and $f_{\lambda, 0}$ acts as multiplication by 2 on the arguments and as the affine contraction $\lambda|z|+1-\lambda$ on radial lines. Also, $f_{\lambda, 0}\left(\mathbb{S}^{1}\right)=\mathbb{S}^{1}$ where $\mathbb{S}^{1}=\{|z|=1\}$. The map $f_{\lambda, c}$ may be written as the postcomposition of $f_{\lambda, 0}$ by the translation $z \mapsto z+c$. Therefore, $f_{\lambda, c}\left(\mathbb{C}^{*}\right)=\{z \in \mathbb{C}|| z \mid>1-\lambda\}+c$.

This family $f_{\lambda, c}$ is closely related to the well known and extensively studied quadratic family $Q_{c}(z)=z^{2}+c$. The action of $Q_{0}$ as $|z|^{2}$ on radial lines has been replaced by an affine contraction. 
Our main interest here will be on maps $f_{\lambda, c}$ for $|c|$ small. For $|c|<1-\lambda$, by the above considerations we have that $f_{c}\left(\mathbb{C}^{*}\right) \subset \mathbb{C}^{*}$. Moreover, it is easy to check that

$$
V=\left\{z \in \mathbb{C}^{*}\left|\exp \left(-\frac{|c|}{1-\lambda}\right)<\right| z \mid<\exp \left(\frac{|c|}{1-\lambda}\right)\right\}
$$

is a trapping region for $f_{\lambda, c}$ when $|c|$ is sufficiently small. That is, $f_{\lambda, c}(\bar{V}) \subset V$. We say that

$$
\Omega_{\lambda, c}=\bigcap_{n \geqslant 0} f_{\lambda, c}^{n}(\bar{V})
$$

is the attractor for the dynamics of $f_{\lambda, c}$.

Proposition 7.2. There exists $\lambda_{0} \in(0,1)$ such that if $\lambda \in\left[\lambda_{0}, 1\right)$, then the attractor of $f_{\lambda, c}$ is homeomorphic to a closed topological annulus for all $c$ in a punctured neighborhood of the origin (which depends on $\lambda$ ).

The proof of this fact relies on considering an appropriate rescaling of $f_{\lambda, c}$ as $c$ goes to 0 . Namely, consider

$$
\begin{aligned}
\iota: \mathbb{R} / \mathbb{Z} \times \mathbb{R} & \rightarrow \mathbb{C}^{*}, \\
(\theta, t) & \mapsto \exp (2 \pi(t+\mathrm{i} \theta))
\end{aligned}
$$

and for $|c|<1-\lambda$, let

$$
\hat{f}_{\lambda, c}=\iota^{-1} \circ f_{\lambda, c} \circ \iota \text {. }
$$

Lemma 7.3. For $\eta>0$ let $h_{\eta}(\theta, t)=(\theta, \eta t)$. Then, for all $\alpha \in \mathbb{R} / \mathbb{Z}$,

$$
h_{\eta}^{-1} \circ \hat{f}_{\lambda, \eta \exp (2 \pi \mathrm{i} \alpha)} \circ h_{\eta}
$$

converges, as $\eta \rightarrow 0$, in the $C^{1}$ topology to:

$$
F_{\lambda, \alpha}(\theta, t)=\left(2 \theta, \lambda t+\frac{1}{2 \pi} \cos 2 \pi(\alpha-2 \theta)\right) .
$$

Proof. Fix $\lambda \in(0,1)$. Let $c(\eta)=\eta \exp (2 \pi \mathrm{i} \alpha)$ and

$$
\left(\Phi_{\eta}(\theta, t), \Psi_{\eta}(\theta, t)\right)=\hat{f}_{\lambda, c(\eta)} \circ h_{\eta}(\theta, t) .
$$

Therefore,

$$
h_{\eta}^{-1} \circ \hat{f}_{\lambda, \eta \exp (2 \pi \mathrm{i} \alpha)} \circ h_{\eta}=\left(\Phi_{\eta}, \frac{\Psi_{\eta}}{\eta}\right) .
$$

We must show that $\Phi_{\eta}(\theta, t) \rightarrow 2 \theta$ and that

$$
\frac{\Psi_{\eta}(\theta, t)}{\eta} \rightarrow \lambda t+(2 \pi)^{-1} \cos (\alpha-2 \theta)
$$

in the $C^{1}$ topology, as $\eta \rightarrow 0$. From $f_{\lambda, c} \circ \iota \circ h_{\eta}=\iota \circ\left(\Phi_{\eta}, \Psi_{\eta} / \eta\right)$ we obtain:

$$
(\lambda \exp (2 \pi \eta t)+1-\lambda) \exp (2 \pi \mathrm{i} 2 \theta)+\eta \exp (2 \pi \mathrm{i} \alpha)=\exp \left(2 \pi\left(\Psi_{\eta}(\theta, t)+\mathrm{i} \Phi_{\eta}(\theta, t)\right)\right) .
$$

It follows that

$$
\exp \left(2 \cdot 2 \pi \mathrm{i}\left(\Phi_{\eta}(\theta, t)-2 \theta\right)\right)=\frac{\lambda \exp (2 \pi \eta t)+1-\lambda+\eta \exp (2 \pi \mathrm{i}(\alpha-2 \theta))}{\lambda \exp (2 \pi \eta t)+1-\lambda+\eta \exp (-2 \pi \mathrm{i}(\alpha-2 \theta))}
$$


converges to the constant function 1 in the $C^{1}$ topology for maps from $\mathbb{R} / \mathbb{Z} \times \mathbb{R}$ into $\mathbb{R}$. Therefore,

$$
\Phi_{\eta}(\theta, t)-2 \theta \rightarrow 0
$$

in the $C^{1}$ topology for maps from $\mathbb{R} / \mathbb{Z} \times \mathbb{R}$ into $\mathbb{R} / \mathbb{Z}$. From (27),

$$
\exp \left(2 \pi \frac{\Psi_{\eta}(\theta, t)}{\eta}\right)=|1+\eta(2 \pi \lambda t+\exp (2 \pi \mathrm{i}(\alpha-2 \theta)+\mathrm{O}(\eta)))|^{1 / \eta},
$$

which, as $\eta \rightarrow 0$, converges to

$$
\exp (2 \pi \lambda t+\cos 2 \pi(\alpha-2 \theta))
$$

in the $C^{1}$ topology. It follows that

$$
\frac{\Psi_{\eta}}{\eta} \rightarrow \lambda t+\frac{1}{2 \pi} \cos 2 \pi(\alpha-2 \theta)
$$

which establishes the lemma.

From Proposition 7.1, there exists $\lambda_{0}$ such that for all $\lambda \in\left[\lambda_{0}, 1\right)$ there exists a neighborhood $\mathcal{U}$ of $\left\{F_{\lambda, \alpha} \mid \alpha \in\right.$ $\mathbb{R} / \mathbb{Z}\} \subset \operatorname{Lip}(\bar{U}, U)$ so that the attractor $\Omega_{F}$ is an annulus for all $F \in \mathcal{U}$ where $U=\mathbb{R} / \mathbb{Z} \times\left(-(1-\lambda)^{-1},(1-\lambda)^{-1}\right)$. Since for $\eta$ sufficiently small, say $0<\eta<\eta_{0}$, and for all $\alpha \in \mathbb{R} / \mathbb{Z}$,

$$
h_{\eta}^{-1} \circ \hat{f}_{\lambda, \eta \exp (2 \pi \mathrm{i} \alpha)} \circ h_{\eta} \in \mathcal{U},
$$

it follows that the attractor set of $\hat{f}_{\lambda, \eta \exp (2 \pi \mathrm{i} \alpha)} \in \operatorname{Lip}(\bar{W}, W)$ is an annulus, where $W=\mathbb{R} / \mathbb{Z} \times\left(-(1-\lambda)^{-1} \eta\right.$, $\left.(1-\lambda)^{-1} \eta\right)$. Therefore, the attractor of $f_{\lambda, \eta \exp (2 \pi \mathrm{i} \alpha)}$ is an annulus for all $0<\eta<\eta_{0}$ and all $\alpha \in \mathbb{R} / \mathbb{Z}$ which proves the claim of Proposition 7.2.

\subsection{With interior and not an annulus}

Proposition 7.4. For all $\lambda>1 / 2$ there exists a $C^{\omega}$ function $\tau: \mathbb{R} / \mathbb{Z} \rightarrow \mathbb{R}$ such that the attractor set $\Omega_{\lambda, \tau}$ of $A_{\lambda, \tau}(\theta, t)=(2 \theta, \lambda t+\tau(\theta))$ has non-empty interior and it is not a topological annulus. Moreover, this property is robust. That is, let $U=\mathbb{R} / \mathbb{Z} \times\left(-T_{0}, T_{0}\right) \subset \mathbb{R} / \mathbb{Z} \times \mathbb{R}$ be such that $A_{\lambda, \tau}(\bar{U}) \subset U$. Then there exists a neighborhood $\mathcal{U}$ of $A_{\lambda, \tau}$ in $\operatorname{Lip}(\bar{U}, U)$ such that for all $F \in \mathcal{U}$ the attractor set $\Omega_{F}$ has non-empty interior and it is not a topological annulus.

A similar example can be constructed for all $\lambda>1 / \ell$ and $\ell>2$. Here we specialize in the case $\ell=2$ for the sake of simplicity of the exposition.

Construction of the example. We endow $\mathbb{R} / \mathbb{Z}$ with its standard orientation and use interval notation accordingly. Let $p \geqslant 1$ be such that $\lambda+\cdots+\lambda^{p-1}>1$ and consider $\eta<1$ such that:

$$
\left(\lambda+\cdots+\lambda^{p-1}\right) \eta>1 .
$$

Consider the periodic cycle $\left\{\theta_{0}=2^{p-1} /\left(2^{p}-1\right), \theta_{1}=m_{2}\left(\theta_{0}\right), \ldots, \theta_{p-1}=m_{2}^{p-1}\left(\theta_{0}\right)\right\}$ of period $p>2$ with subindices mod $p$. Observe that the subindices respect cyclic order and that the interval $I_{0}=\left(\theta_{0}, \theta_{1}\right)$ has length $\left|I_{0}\right|>1 / 2$.

Consider positive constants $T_{0}, T_{1}, \delta, \epsilon_{0}, \ldots, \epsilon_{p-1}, \lambda^{\prime}$ and a natural number $N \geqslant 2$ such that

$$
\begin{aligned}
& T_{0}>\frac{1}{1-\lambda}, \\
& \frac{T_{0}}{\lambda}>\frac{\lambda-\lambda^{N}}{1-\lambda}+\frac{\lambda^{N} T_{0}}{1-\lambda}=T_{1},
\end{aligned}
$$




$$
\begin{aligned}
& \delta=\left|I_{0}\right|-1 / 2, \\
& 0<\epsilon_{0}<\delta \cdot 2^{-N+1}, \\
& 2 \epsilon_{j}<\epsilon_{j+1} \quad \text { for } j=1, \ldots, p-1, \\
& \lambda \eta<\lambda^{\prime}<\lambda .
\end{aligned}
$$

Let $\tau: \mathbb{R} / \mathbb{Z} \rightarrow \mathbb{R}$ be a $C^{\infty}$ function such that:

$$
\begin{aligned}
& \tau(\theta)=0 \quad \text { for all } \theta \in I_{0}^{\epsilon_{0}}, \\
& \tau(\theta)=\lambda^{\prime} \quad \text { for all } \theta \in I_{j}^{\epsilon_{j}} \quad \text { and } j=1, \ldots, p-1
\end{aligned}
$$

where $I_{j}^{\epsilon_{j}}=\left(\theta_{j}+\epsilon_{j}, \theta_{j+1}-\epsilon_{j}\right)$,

$$
\begin{aligned}
& \tau\left(\theta_{j}\right)=T_{0} \quad \text { for } j=0,1, \ldots, p-1, \\
& 0<\tau(\theta) \leqslant T_{0} \quad \text { for } \theta \in \mathbb{R} / \mathbb{Z} \backslash I_{0} .
\end{aligned}
$$

The next three lemmas are devoted to prove that the properties stated in Proposition 7.4 hold for a function $\tau: \mathbb{R} / \mathbb{Z} \rightarrow \mathbb{R}$ as above. It follows that there is a Lipschitz close $C^{\omega}$ function for which the statement of the proposition holds.

Lemma 7.5. Let $\rho^{ \pm}=\rho_{A_{\lambda, \tau}}^{ \pm}$on $\mathbb{R} / \mathbb{Z}$. Then

(1) $\rho^{-}(\theta)=0$ for all $\theta \in \mathbb{R} / \mathbb{Z}$.

(2) $\rho^{+}\left(\theta_{j}\right)=(1-\lambda)^{-1} T_{0}$ for all $j=0, \ldots, p-1$.

(3) $\rho^{+}\left(\theta_{0}+\frac{1}{2}\right)<\lambda^{-1} T_{0}$.

Proof. (1) Since

$$
\left|I_{0}^{\epsilon_{0}}\right|=\left|I_{0}\right|-2 \epsilon_{0}>\left|I_{0}\right|-2 \delta \cdot 2^{-N+1} \geqslant\left|I_{0}\right|-\delta,
$$

it follows that $\mathbf{m}_{2}\left(I_{0}^{\epsilon_{0}}\right)=\mathbb{R} / \mathbb{Z}$. Therefore, given $\theta_{0}^{\prime} \in \mathbb{R} / \mathbb{Z}$ and $n \geqslant 1$ there exists $\theta_{n}^{\prime} \in I_{0}^{\epsilon_{0}}$ such that $2 \theta_{n}^{\prime}=\theta_{n-1}^{\prime}$. Hence, $\rho^{-}\left(\theta_{0}^{\prime}\right) \leqslant t_{\lambda}\left(\left(\theta_{n}^{\prime}\right)\right)=0$ (see Proposition 2.5). Also $0 \leqslant \rho^{-}\left(\theta_{0}^{\prime}\right)$ since $\tau \geqslant 0$.

(2) Since $\tau \leqslant T_{0}$, it follows that $\rho^{+} \leqslant(1-\lambda)^{-1} T_{0}$. Now $\rho^{+}\left(\theta_{j}\right)=(1-\lambda)^{-1} T_{0}$ because each one of the periodic points $\theta_{j}$ has as an infinite backward orbit along the periodic orbit $\theta_{0}, \ldots, \theta_{p-1}$.

(3) Let $\theta_{0}^{\prime}=\theta_{0}+1 / 2$ and $\left(\theta_{n}^{\prime}\right) \in \mathcal{S}$ be a backward orbit. Let

$$
N_{0}=\min \left\{n \mid \theta_{n}^{\prime} \notin I_{0}^{\epsilon_{0}} \cup \cdots \cup I_{p-1}^{\epsilon_{p-1}}\right\} .
$$

Then

$$
\operatorname{dist}_{\mathbb{R} / \mathbb{Z}}\left(\theta_{N_{0}}^{\prime},\left\{\theta_{j}\right\}_{0}^{p-1}\right) \leqslant \max \left\{\epsilon_{j}\right\}=\epsilon_{0}<\delta \cdot 2^{-N+1} .
$$

Hence,

$$
\delta=\operatorname{dist}_{\mathbb{R} / \mathbb{Z}}\left(\theta_{0}^{\prime},\left\{\theta_{j}\right\}_{0}^{p-1}\right)<\delta \cdot 2^{-N+1} \cdot 2^{N_{0}} .
$$

We conclude that $N_{0} \geqslant N$ and

$$
\begin{aligned}
\rho^{+}\left(\theta_{0}^{\prime}\right) & \leqslant \tau\left(\theta_{1}^{\prime}\right)+\lambda \tau\left(\theta_{2}^{\prime}\right)+\cdots+\lambda^{N_{0}-2} \tau\left(\theta_{N_{0}-1}^{\prime}\right)+\frac{\lambda^{N_{0}} T_{0}}{1-\lambda} \leqslant \lambda+\lambda^{2}+\cdots+\lambda^{N_{0}-1}+\frac{\lambda^{N_{0}} T_{0}}{1-\lambda} \\
& =\frac{\lambda-\lambda^{N_{0}}}{1-\lambda}+\frac{\lambda^{N_{0}} T_{0}}{1-\lambda} \leqslant \frac{\lambda-\lambda^{N}}{1-\lambda}+\frac{\lambda^{N} T_{0}}{1-\lambda}<\lambda^{-1} T_{0} .
\end{aligned}
$$


Lemma 7.6. There exists an open neighborhood $\mathcal{U}$ of $A_{\lambda, \tau}$ in $\operatorname{Lip}(\bar{U}, U)$ such that for all $F \in \mathcal{U}$ the attractor $\Omega_{F}$ is not a closed topological annulus.

Proof. If we denote by $\gamma^{ \pm}$the graphs of $\rho_{A_{\lambda, \tau}}^{ \pm}$, then

$$
\begin{aligned}
\min \left\{t \mid\left(\theta_{1}, t\right) \in A_{\lambda, \tau}\left(\gamma^{+}\right)\right\} & =\min \left\{\lambda \rho^{+}\left(\theta_{0}+1 / 2\right)+\tau\left(\theta_{0}+1 / 2\right), \lambda \rho^{+}\left(\theta_{0}\right)+\tau\left(\theta_{0}\right)\right\} \\
& =\min \left\{\lambda \rho^{+}\left(\theta_{0}+1 / 2\right),(1-\lambda)^{-1} T_{0}\right\} \\
& <T_{0} \\
& =\max \left\{0, T_{0}\right\} \\
& =\max \left\{\lambda \rho^{-}\left(\theta_{0}+1 / 2\right)+\tau\left(\theta_{0}+1 / 2\right), \lambda \rho^{-}\left(\theta_{0}\right)+\tau\left(\theta_{0}\right)\right\} \\
& =\max \left\{t \mid\left(\theta_{1}, t\right) \in A_{\lambda, \tau}\left(\gamma^{-}\right)\right\} .
\end{aligned}
$$

By Lemma 6.8, it follows that for all $F$ close to $A_{\lambda, \tau}$ the attractor $\Omega_{F}$ is not an annulus.

Lemma 7.7. There exists an open neighborhood $\mathcal{U}$ of $A_{\lambda, \tau}$ in $\operatorname{Lip}(\bar{U}, U)$ such that for all $F \in \mathcal{U}$ the attractor $\Omega_{F}$ has non-empty interior.

Proof. Let $\eta^{\prime}$ be such that

$$
\left(\lambda+\cdots+\lambda^{p-1}\right) \eta>\eta^{\prime}>1 .
$$

By Proposition 6.2 we may assume that for all $F$ sufficiently close to $A_{\lambda, \tau}$ the lower boundary $\rho_{F}^{-}: \mathbb{R} / \mathbb{Z} \rightarrow \mathbb{R}$ is well defined and

$$
\rho_{F}^{-}<\left(\lambda^{\prime}\right)^{2} \text {. }
$$

Consider the subsets $R_{1}^{F}$ and $R_{2}$ of $\mathbb{R} / \mathbb{Z} \times \mathbb{R}$ defined by:

$$
\begin{aligned}
& R_{1}^{F}=\left\{(\theta, t) \mid \rho_{F}^{-}(\theta) \leqslant t \leqslant \lambda\right\} \\
& R_{2}=\overline{I_{0}^{\epsilon_{0}}} \times\left[\lambda, \eta^{\prime}\right] \cup \bigcup_{j=2}^{p-1}\left(\overline{I_{j}^{\epsilon_{j}}} \times\left[\lambda,\left(\lambda+\cdots+\lambda^{j}\right) \eta\right]\right) .
\end{aligned}
$$

Let $R^{F}=R_{1}^{F} \cup R_{2}$. We will show that the $F\left(R^{F}\right) \supset R^{F}$ for all $F$ in a sufficiently small neighborhood of $A_{\lambda, \tau}$.

To simplify notation, let $R=R^{A_{\lambda, \tau}}$. We start by showing that $A_{\lambda, \tau}($ int $R) \supset R_{2}$. In fact, since for $j=1, \ldots$, $p-1$

$$
I_{j}^{\epsilon_{j}} \times\left(0,\left(\lambda+\cdots+\lambda^{j}\right) \eta\right) \subset \operatorname{int} R
$$

and $\tau\left(I_{j}^{\epsilon_{j}}\right)=\left\{\lambda^{\prime}\right\}$, it follows that

$$
\mathbf{m}_{2}\left(I_{j}^{\epsilon_{j}}\right) \times\left(\lambda^{\prime}+\lambda\left(\lambda+\cdots+\lambda^{j}\right) \eta\right) \subset A_{\lambda, \tau}(\text { int } R) .
$$

By (34), $\mathbf{m}_{2}\left(I_{j}^{\epsilon_{j}}\right) \supset \overline{I_{j+1}^{\epsilon_{j+1}}}$ and, by (35), $\left(\lambda^{\prime}+\lambda\left(\lambda+\cdots \lambda^{j}\right) \eta\right) \supset\left[\lambda, \lambda \eta+\lambda\left(\lambda+\cdots+\lambda^{j}\right) \eta\right]$. Therefore, $A_{\lambda, \tau}($ int $R) \supset$ $R_{2}$. Hence, for all $F$ sufficiently close to $A_{\lambda, \tau}$ we also have that

$$
F\left(\text { int } R^{F}\right) \supset R_{2}
$$

since the boundaries of $R^{F}$ move continuously with $F$.

For $F$ sufficiently close to $A_{\lambda, \tau}$ the circle $\mathbb{R} / \mathbb{Z} \times\{\lambda\}$ has image contained in $t>\lambda^{\prime} \lambda$. Therefore,

$$
F\left\{(\theta, t) \mid \rho_{F}^{-}(\theta) \leqslant t \leqslant \lambda\right\} \supset\left\{(\theta, t) \mid \rho_{F}^{-}(\theta) \leqslant t \leqslant \lambda \lambda^{\prime}\right\} .
$$


Since $A_{\lambda, \tau}\left(I_{0}^{\epsilon_{0}} \times\left(\lambda \eta, \eta^{\prime}\right)\right) \supset \mathbb{R} / \mathbb{Z} \times\left(\lambda \lambda^{\prime} \eta, \lambda \eta^{\prime}\right) \supset \mathbb{R} / \mathbb{Z} \times\left[\lambda \lambda^{\prime}, \lambda\right]$, for all $F$ sufficiently close to $A_{\lambda, \tau}$ we also have that

$$
F\left(R^{F}\right) \supset \mathbb{R} / \mathbb{Z} \times\left[\lambda \lambda^{\prime}, \lambda\right] .
$$

From (41) and (42) we conclude that $F\left(R^{F}\right) \supset R_{1}^{F}$. By (40), we obtain that $F\left(R^{F}\right) \supset R^{F}$.

Since for all $F$ sufficiently close to $A_{\lambda, \tau}$ we have that $F\left(R^{F}\right) \supset R^{F}$, it follows that $R^{F} \subset \Omega_{F}$ and therefore $\Omega_{F}$ has non-empty interior.

\section{Acknowledgements}

Juan Rivera-Letelier is grateful to J.-C. Yoccoz for useful conversations and valuable comments and Rodrigo Bamón thanks Marcelo Viana for helpful and constructive discussions. The authors appreciate discussions with Godofredo Iommi.

\section{References}

[1] T. Bousch, La condition de Walters, Ann. Sci. École Norm. Sup. 34 (2001) 287-311.

[2] G. Contreras, A.O. Lopes, Ph. Thieullen, Lyapunov minimizing measures for expanding maps of the circle, Ergodic Theory Dynam. Systems 21 (2001) 1379-1409.

[3] V.A. Dobrynskiı̌, There exist endomorphisms of a plane that have two-dimensional attractors, Dokl. Akad. Nauk 364 (1999) $303-305$.

[4] P. Frederickson, J.L. Kaplan, E.D. Yorke, J.A. Yorke, The Liapunov dimension of strange attractors, J. Differential Equations 49 (1983) 185-207.

[5] O. Jenkinson, R.D. Mauldin, M. Urbański, Ergodic optimization for non-compact dynamical systems, preprint, September 2004.

[6] J.L. Kaplan, J.A. Yorke, Chaotic behavior of multidimensional difference equations, in: Functional differential equations and approximation of fixed points, Proc. Summer School and Conf., Univ. Bonn, Bonn, 1978, in: Lecture Notes in Math., vol. 730, Springer, Berlin, 1979, pp. 204-227.

[7] Y. Katznelson, An Introduction to Harmonic Analysis, Dover, 1976.

[8] J. Milnor, On the concept of attractor, Comm. Math. Phys. 99 (1985) 177-195.

[9] F. Pryzyticki, On $U$-stability and structural stability of endomorphisms satisfying Axiom A, Studia Math. 60 (1977) 61-77.

[10] M. Tsujii, Fat solenoidal attractors, Nonlinearity 14 (2001) 1011-1027.

[11] M. Tsujii, Physical measures for partially hyperbolic surface endomorphisms, math.DS/0301243.

[12] M. Viana, Multidimensional nonhyperbolic attractors, Inst. Hautes Études Sci. Publ. Math. 85 (1997) $63-96$. 